\title{
Textural and geochemical characteristics of (1) comasuat late Pleistocene to Holocene fine-grained deep-sea sediment cores (GM6 and GM7), recovered from southwestern Gulf of Mexico
}

\author{
Abigail Anaya-Gregorio ${ }^{1}$, John S. Armstrong-Altrin ${ }^{2 *}$, María Luisa Machain-Castillo², Patricia C. Montiel-García ${ }^{3}$ \\ and Mayla A. Ramos-Vázquez ${ }^{4}$
}

\begin{abstract}
Texture, mineralogy, geochemistry, and ${ }^{14} \mathrm{C}$ ages of two deep-sea sediment cores (GM6 and GM7) recovered in the southwestern Gulf of Mexico were investigated to infer their provenance and depositional condition. The sediments are enriched in fine-grained silt and clay and poor in sand content. Mineralogically, the sediments consist of quartz, calcite, smectite, and kaolinite. Based on the ${ }^{14} \mathrm{C}$ data, the age of the GM6 and GM7 sediment cores were calculated as 23,615 cal yrs. BP and 19,007 cal yrs. BP, respectively. The weathering indices such as chemical index of alteration (CIA), chemical index of weathering (CIM), and plagioclase index of alteration (PIA) in GM6 (56-69, 60-74, and 61-73, respectively) and GM7 (54-69, 57-76, and 55-74, respectively) cores revealed a moderate-to-high intensity of weathering. The rare earth element (REE) patterns suggested that the sediments were derived mostly by the weathering of intermediate rocks, exposed along the coastal regions of the Gulf of Mexico. The trace elemental ratios like $\mathrm{V} / \mathrm{Cr}(<2)$, $\mathrm{Ni} / \mathrm{Co}(<2)$, and $\mathrm{Cu} / \mathrm{Zn}(<1)$, authigenic uranium content $(<1)$, and $\mathrm{Ce}$ anomaly $\left(\mathrm{Ce} / \mathrm{Ce}^{*} \leq 1\right)$ suggested that the sediments were deposited under an oxic condition, which was also revealed by the benthic foraminiferal assemblages throughout the GM6 and GM7 sediment cores.
\end{abstract}

Keywords: Radiocarbon age, Manganosite, Weathering, Palaeoredox condition, Provenance, Holocene, Gulf of Mexico

\section{Introduction}

The composition of terrigenous sediments is largely influenced by the nature of parent rocks and sedimentary processes during transportation and within the depositional basin (Armstrong-Altrin 2015; Basu 2017). Hence, the spatial and temporal variations during sedimentation can be able to understand by the mineralogical and geochemical compositions of sediments, and numerous studies utilized these as a device to understand sediment provenance (Bhattacharjee et al. 2017; Craigie 2018; Hou et al. 2017; Mitra et al. 2017; Paikaray et al. 2008; Saha et al. 2010,

\footnotetext{
*Correspondence: armstrong@cmarl.unam.mx; john_arms@yahoo.com ${ }^{2}$ Instituto de Ciencias del Mar y Limnología, Universidad Nacional Autónoma de México, Unidad de Procesos Oceánicos y Costeros, Ciudad Universitaria, 04510 Ciudad de México, Mexico

Full list of author information is available at the end of the article
}

2018; Verma et al. 2016a; Verma and Armstrong-Altrin 2016; Wang et al. 2017a, 2017b). In sediments, unstable minerals and mobile elements can be affected easily by weathering and erosional processes during transportation. However, immobile elements like REE, Co, Cr, Th, and Sc in sediments are considered as good provenance indicators, because they are not fractionated during sedimentary processes like diagenesis and weathering (Fedo et al. 1995). The fine-grained detrital sediments are particularly helpful for provenance study, because, they provide new insights into the sedimentation processes through geological time (Ramos-Vázquez et al. 2017; Selvaraj et al. 2015).

Previous studies by Carranza-Edwards et al. (2001) reported the composition of coastal sediments from the northwestern part of the Gulf of Mexico and inferred their provenance. Recently, Armstrong-Altrin et al. (2015a) and 
Hernández-Hinojosa et al. (2018) investigated the origin of coastal sediments in the Veracruz and Tabasco states, Gulf of Mexico. Other studies documented the textural characteristics (Kasper-Zubillaga et al. 2013), heavy metal concentrations and geochemistry of estuary (Rosales-Hoz et al. 2015) and continental slope sediments (Armstrong-Altrin et al. 2015b; Ramos-Vázquez et al. 2017) from the western part of the Gulf of Mexico. Although numerous researchers investigated the provenance of coastal sediments along the Gulf of Mexico, studies concentrated on the textural and geochemical characteristics of fine-grained sediments in the southwestern Gulf of Mexico are little. In the present study, we document texture, mineralogy, geochemistry, and ${ }^{14} \mathrm{C}$ age data of two fine-grained sediment cores (GM6 and GM7) retrieved in the southwestern Gulf of Mexico. The aims of this investigation are to investigate the chemical weathering, source rock composition, radiocarbon age, and the depositional condition.

\section{Study area}

Sampling of two sediment cores GM6 (Latitude 19 $08^{\prime}$ 64.54" $\mathrm{N}$ and Longitude $\left.93^{\circ} 59^{\prime} 81.90^{\prime \prime} \mathrm{W}\right)$ and GM7 (Latitude $19^{\circ} 12^{\prime} 37.45^{\prime \prime} \mathrm{N}$ and Longitude $94^{\circ} 06^{\prime} 13.15^{\prime \prime} \mathrm{W}$ ) (Fig. 1) was performed in the southwestern part of the

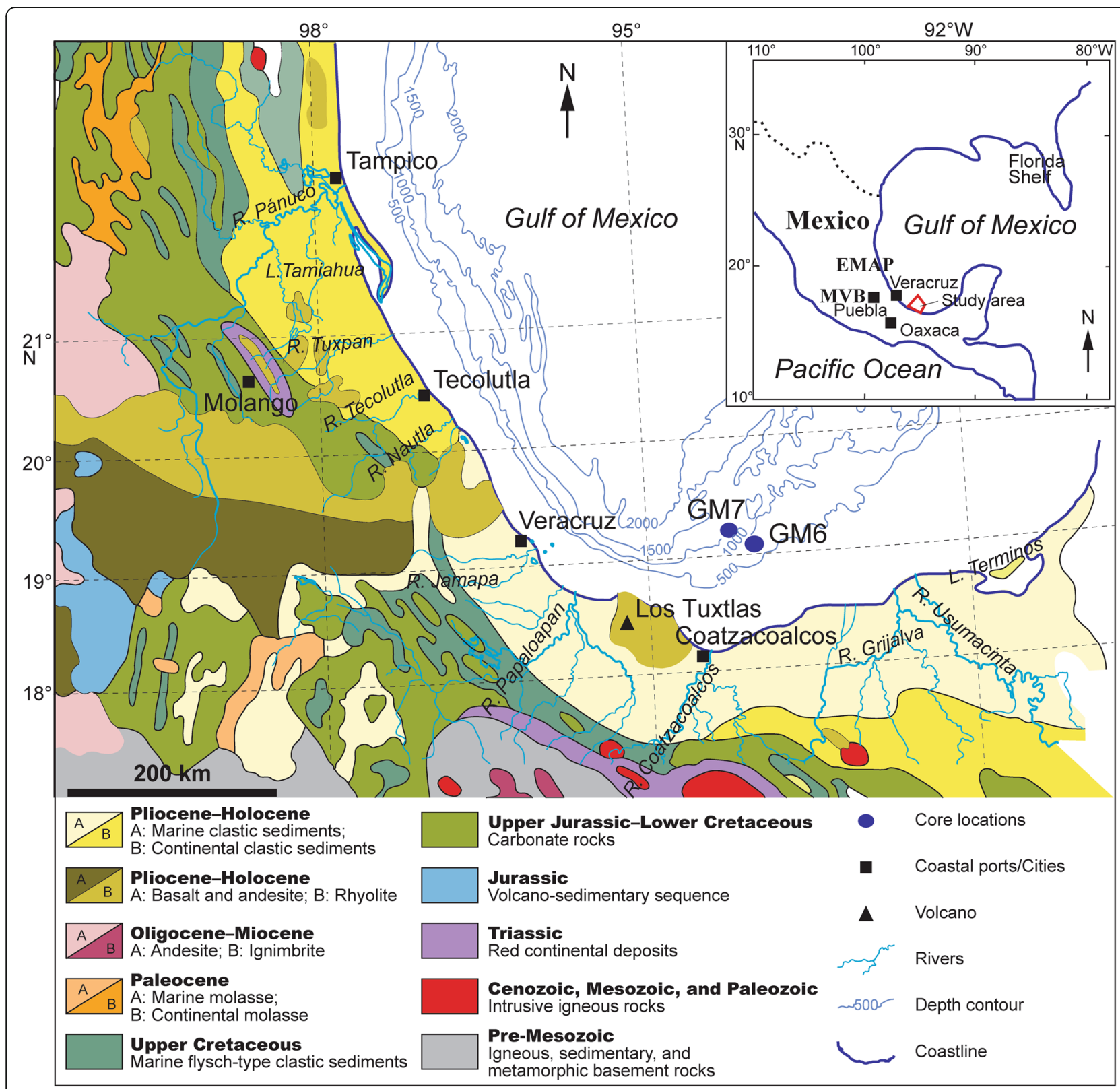

Fig. 1 Map showing core locations and geology of the Gulf of Mexico coastal areas (Source: Carta Geológica; scale 1:1,000,000) (modified after Armstrong-Altrin 2015). MVB = Mexican Volcanic Belt; EMAP = Eastern Mexican Alkaline Province 
Table 1 Grain size and textural parameters (Folk 1980) for the deep-sea sediments of the southwestern Gulf of Mexico

\begin{tabular}{|c|c|c|c|c|c|c|c|c|c|c|c|c|}
\hline Core No. & \multicolumn{6}{|l|}{ GM6 } & \multicolumn{6}{|l|}{ GM7 } \\
\hline Water depth & \multicolumn{6}{|c|}{$856 \mathrm{~m}$} & \multicolumn{6}{|c|}{$989 \mathrm{~m}$} \\
\hline Latitude & \multicolumn{6}{|c|}{$19^{\circ} 08^{\prime} 64.54 " \mathrm{~N}$} & \multicolumn{6}{|c|}{$19^{\circ} 12^{\prime} 37.45^{\prime \prime} \mathrm{N}$} \\
\hline Longitude & \multicolumn{6}{|c|}{$93^{\circ} 59^{\prime} 81.90^{\prime \prime} \mathrm{W}$} & \multicolumn{6}{|c|}{$94^{\circ} 06^{\prime} 13.15^{\prime \prime} \mathrm{W}$} \\
\hline Section (cm) & $0-1$ & $91-92$ & $151-152$ & $222-223$ & $300-301$ & Mean $\pm 1 \mathrm{~s}(n=5)$ & $0-1$ & $91-92$ & $151-152$ & $222-223$ & $300-301$ & Mean $\pm 1 \mathrm{~s}(n=5)$ \\
\hline Sand (\%) & 0.02 & 0.11 & - & 0.089 & 0.008 & $0.04 \pm 0.05$ & - & - & - & 0.299 & 0.003 & $0.06 \pm 0.13$ \\
\hline Silt (\%) & 52.3 & 50.9 & 48.2 & 49.4 & 51.5 & $50.5 \pm 1.67$ & 49.9 & 46.3 & 49.4 & 54.4 & 48.6 & $49.7 \pm 2.93$ \\
\hline Clay (\%) & 47.7 & 48.9 & 51.9 & 50.5 & 48.5 & $49.5 \pm 1.67$ & 50.2 & 53.7 & 50.7 & 45.4 & 51.4 & $50.3 \pm 3.05$ \\
\hline $\mathrm{Mz}(\varphi)$ & 7.99 & 8.02 & 8.13 & 8.06 & 7.99 & $8.04 \pm 0.06$ & 8.07 & 8.17 & 7.99 & 7.80 & 8.07 & $8.02 \pm 0.14$ \\
\hline Sorting $(\sigma \mid \varphi)$ & 1.56 & 1.59 & 1.53 & 1.60 & 1.58 & $1.57 \pm 0.03$ & 1.54 & 1.57 & 1.73 & 1.74 & 1.65 & $1.64 \pm 0.09$ \\
\hline Skewness $\left(\mathrm{Sk}_{1}\right)$ & 0.05 & 0.02 & 0.02 & 0.00 & 0.02 & $0.02 \pm 0.01$ & 0.03 & 0.02 & 0.07 & 0.02 & 0.03 & $0.03 \pm 0.02$ \\
\hline Kurtosis $\left(K_{G}\right)$ & 0.93 & 0.94 & 0.92 & 0.94 & 0.92 & $0.93 \pm 0.01$ & 0.93 & 0.95 & 0.93 & 0.94 & 0.93 & $0.94 \pm 0.01$ \\
\hline
\end{tabular}

Gulf of Mexico, during a cruise program by a research vessel "Justo Sierra".

\section{Geological backgrounds}

A brief description about the rivers traversing along the southwestern Mexico and the geological setting of the study area is given below:

The Usumacinta and Grijalva Rivers, $1100 \mathrm{~km}$ and $640 \mathrm{~km}$ long, respectively, are the longest rivers in Mexico, that discharges annually $\sim 2678 \mathrm{~m}^{3} \mathrm{~s}^{-1}$, with peak discharges from July to November and rank second in fresh water discharge to the Gulf of Mexico (Day et al. 2003; Salas-de-León et al. 2008). These rivers originate from the northwestern part of the Guatemala and Chiapas (Mexico), respectively, and join together just $\sim 50 \mathrm{~km}$ upstream the coast of the Gulf of Mexico (Armstrong-Altrin et al. 2017).

The Coatzacoalcos River originates from the Veracruz and Oaxaca States and drains between $17^{\circ} 46^{\prime} \mathrm{N}-92^{\circ} 25^{\prime}$ $\mathrm{W}$ and $18^{\circ} 10^{\prime} \mathrm{N}-94^{\circ} 31^{\prime} \mathrm{W}$. The Coatzacoalcos River lithofacies type consists of metasedimentary and volcanic rocks of the Oaxacan Complex. The Oaxacan Complex is located in the north of the Oaxaca State, which represents the most extensive outcrop of $\sim 1 \mathrm{Ga}$ basement in southern Mexico $\left(\sim 10,000 \mathrm{~km}^{2}\right)$. It is composed of anorthosite, garnet-bearing charnockite, gabbros (U$\mathrm{Pb}$ age of $\sim 1230 \mathrm{Ma})$, diorite $(\sim 1012 \mathrm{Ma})$, and syenite
( $1130 \mathrm{Ma})$ (Solari et al. 2007). In general, the lithology of the Gulf of Mexico coastal area was dominated by alluvium (Quaternary), andesite and basalt (Cenozoic), sandstone and shale (Mesozoic), and schist and gneiss (Paleozoic) (Verma et al. 2016b) (Fig. 1).

\section{Materials and methods}

The 5.1 and 4.8-m-long sediment cores (GM6 and GM7) were recovered at 856 and $989 \mathrm{~m}$ water depth, respectively, at the southwestern Gulf of Mexico (Fig. 1). Forty samples at several intervals were selected for various analyses to deduce sediment provenance (Table 1).

Petrographic analysis was performed on 20 thinsections to realize sediment composition. The grain-size and mineralogy were determined by Beckman Coulter particle size analyzer and Siemens D5000 X-ray Diffractometer (XRD), respectively, located at the Sedimentology Laboratory, Universidad Nacional Autónoma de México (UNAM). In addition, the chemistry of selected minerals was determined by a PHILLIPS XL-30 scanning electron microscope (SEM) equipped with energy dispersive spectrometer (EDS) at UNAM, Mexico.

For geochemical analysis, $20 \mathrm{~g}$ of 40 sediment samples were powdered $(<62 \mu \mathrm{m})$ by an agate mortar. Bulksediment major-element concentrations were determined by X-ray fluorescence analysis (XRF) on fused

Table $2{ }^{14} \mathrm{C}$ AMS ages for mixed planktic foraminifers

\begin{tabular}{|c|c|c|c|c|c|c|}
\hline Core No. & Core section $(\mathrm{cm})$ & Species & ${ }^{14} \mathrm{C}$ AMS age (yrs) & $\begin{array}{l}{ }^{14} \mathrm{C} \text { error } \\
( \pm \text { year) }\end{array}$ & $\begin{array}{l}\text { Calibrated age } \\
\text { (cal yrs. BP) }\end{array}$ & $\begin{array}{l}\text { Calibrated erro } \\
( \pm \text { year, } 1 \text { б) }\end{array}$ \\
\hline \multirow[t]{5}{*}{ GM6 } & $60-61$ & Mixed planktic foraminifers & 2660 & 36 & 2388 & 51 \\
\hline & $90-91$ & & 4400 & 36 & 4603 & 76 \\
\hline & $150-151$ & & 7030 & 44 & 7544 & 43 \\
\hline & 190-191 & & 9170 & 44 & 10,021 & 85 \\
\hline & $300-301$ & & 20,120 & 82 & 23,615 & 157 \\
\hline \multirow[t]{2}{*}{ GM7 } & $100-101$ & & 6790 & 44 & 7346 & 47 \\
\hline & $210-211$ & & 16,190 & 73 & 19,007 & 218 \\
\hline
\end{tabular}


glass beads at the geochemistry laboratory at UNAM, Mexico. The analytical accuracy is better than 5\%. A detail about the analytical procedure and standards was described in Lozano and Bernal (2005). The trace and rare earth element concentrations were quantified using an inductively coupled plasma mass spectrometer (ICP-MS), following a methodology provided by Jarvis (1988). The accuracy, measured by an international standard BCR-2 was generally within $10 \%$.

Samples for foraminiferal analysis were wet sieved through a 60 ASTM mesh $(0.25 \mathrm{~mm})$. Aliquots of the washed residues containing 300-500 foraminiferal individuals were analyzed through the microscope, and the specimens were taxonomically determined and counted. Separate fractions were used for benthic and planktic foraminifers. Benthic foraminifers were picked from the $\geq 63 \mu \mathrm{m}$ fraction and planktonic from the $\geq 150 \mu \mathrm{m}$. Planktonic foraminifers were used to construct a biostratigraphic framework and benthics were analyzed for hypoxic characteristics.

Chronostratigraphic framework was achieved by ${ }^{14} \mathrm{C}$ dating on 20 micrograms of foraminiferal species Globigerinoides ruber, sent to Beta Analytics for accelerator mass spectrometry (AMS) determination. The data were converted to calendar years with the CALIB program (Stuiver and Reimer 1993, version 502; http://calib.qub. ac.uk) (Table 2).

\section{Results}

\subsection{Time framework}

The time framework was elaborated based on seven ${ }^{14} \mathrm{C}$ dates, which was followed by the biostratigraphical scheme of Kennett and Huddlestun (1972) and Kennett et al. (1985). The bottom section of core GM6 reaches 23,615 cal yrs. BP (subzone Y2), with the limit of Pleistocene/Holocene at around $195 \mathrm{~cm}$ interval. Core GM7 bottom section revealed a ${ }^{14} \mathrm{C}$ age of about $19,007 \mathrm{cal}$ yrs. $\mathrm{BP}$, also fall within the subzone Y2. The Pleistocene/ Holocene boundary is identified at $130 \mathrm{~cm}$ interval (Table 2, Fig. 2).

\subsection{Granulometric analysis}

The textural characteristics were studied at five intervals for the GM6 and GM7 cores (Table 1). The sediments are enriched in silt and clay contents, and poor in sand content in the cores GM6 (50.5 $\pm 1.67,49.5 \pm 1.67$, and

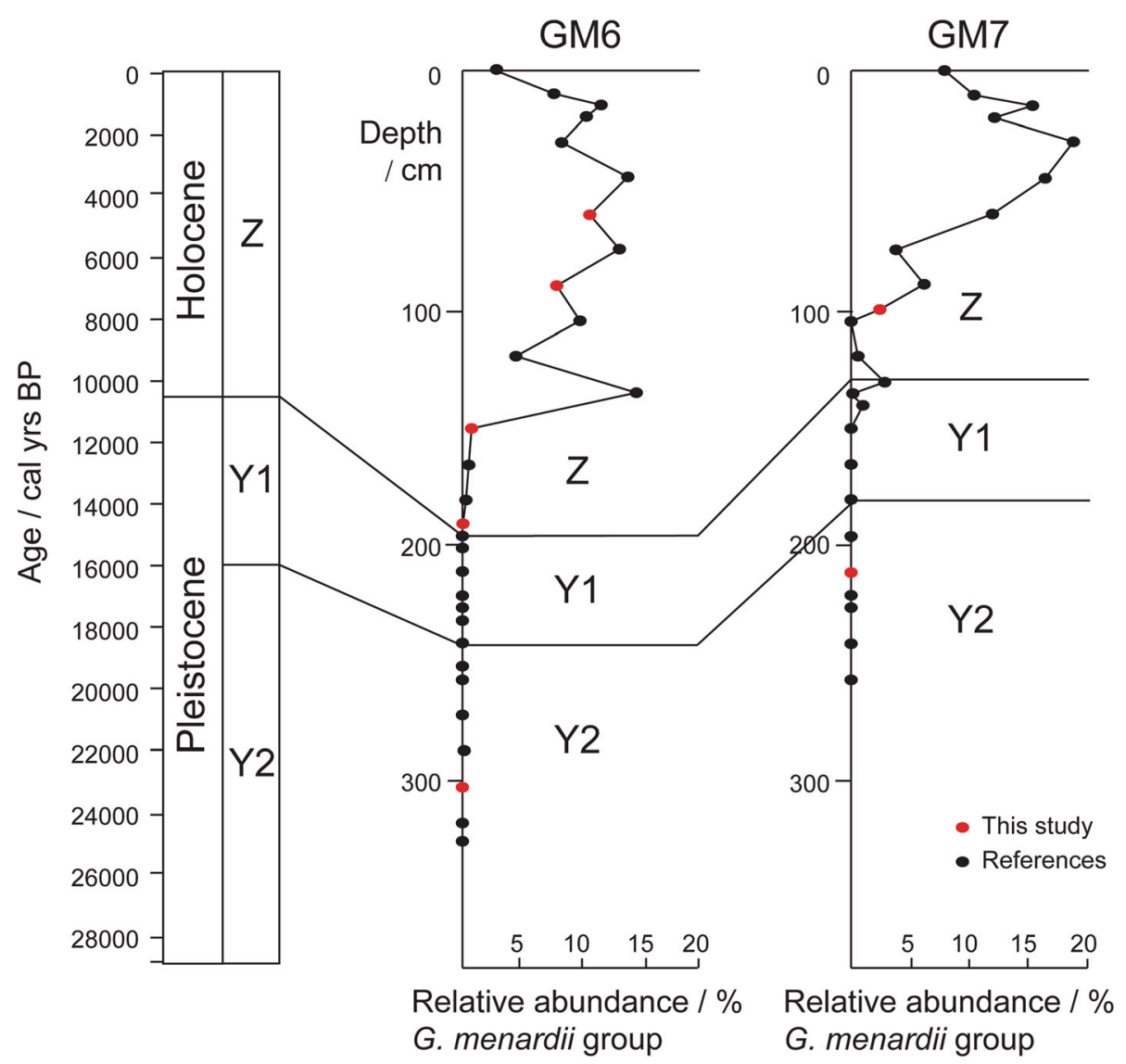

Fig. 2 Biostratigraphic correlation and ${ }^{14} \mathrm{C}$ ages (cal yrs. BP) for the sediment cores. The zone $(Y)$ and sub-zones (Y1, and $\left.Y_{2}\right)$ are based on the biostratigraphic classification of Kennett and Huddlestun (1972) and Kennett et al. (1985) 
$0.04 \pm 0.05$, respectively) and GM7 $(49.7 \pm 2.93,50.3 \pm$ 3.05 , and $0.06 \pm 0.13$, respectively) (Table 1 ). The mean grain size $(\mathrm{Mz})$ is similar for the GM6 and GM7 cores, which ranges from $7.99 \phi$ to $8.13 \phi$ and $7.80 \phi$ to $8.17 \phi$, respectively, indicating that the sediments were dominated by silt and clay. The standard deviation values vary between $1.53-1.60$ and 1.54-1.74 $\phi$ in the GM6 and GM7 cores, respectively, which reveal that the sediments are poorly sorted. The skewness $\left(\mathrm{Sk}_{1}\right)$ values vary between $0.02-0.05$ and $0.02-0.07 \phi$ for the GM6 and GM7 cores, respectively, indicating that the energy was constant during the deposition of sediments. The kurtosis $\left(\mathrm{K}_{\mathrm{G}}\right)$ values of the GM6 and GM7 cores (0.92-0.94 and 0.93-0.95, respectively) indicate that the sediments are mesokurtic type (Table 1 ).

\subsection{Mineralogy}

The X-ray diffraction (XRD) data reveal that quartz and calcite are the major minerals, and muscovite, anorthite, halite, diopside, fayalite, and pyrite are the minor minerals in core GM6 (Fig. 3a; 300-301 cm interval; Last glacial maximum, subzone Y2). On the other hand, in core GM7, quartz and calcite are identified as major, and halite, albite, magnetite, aragonite, and manganosite as minor minerals (Fig. 3c; 290-291 cm interval). Smectite and kaolinite are the dominant clay minerals in both cores (Fig. $3 b$ and d).

The SEM energy dispersive spectrometer (SEM-EDS) analysis of sediments on selected intervals of the cores GM6 and GM7 reveals the concentration of minerals such as, plagioclase, halite, pyrite, and biogenic residues (Fig. 4). The minerals identified by SEM-EDS are consistent with the minerals identified by XRD method.

\subsection{Major element concentrations}

The variations in major element concentrations between the GM6 and GM7 cores, as well as its vertical distributions, are not statistically significant (Table 3). The upper continental crust (UCC; Taylor and McLennan 1985) normalized major element patterns in the two cores are similar, except $\mathrm{MnO}$ content, which is slightly higher in few samples at different intervals of cores GM6 and GM7 (Fig. 5).

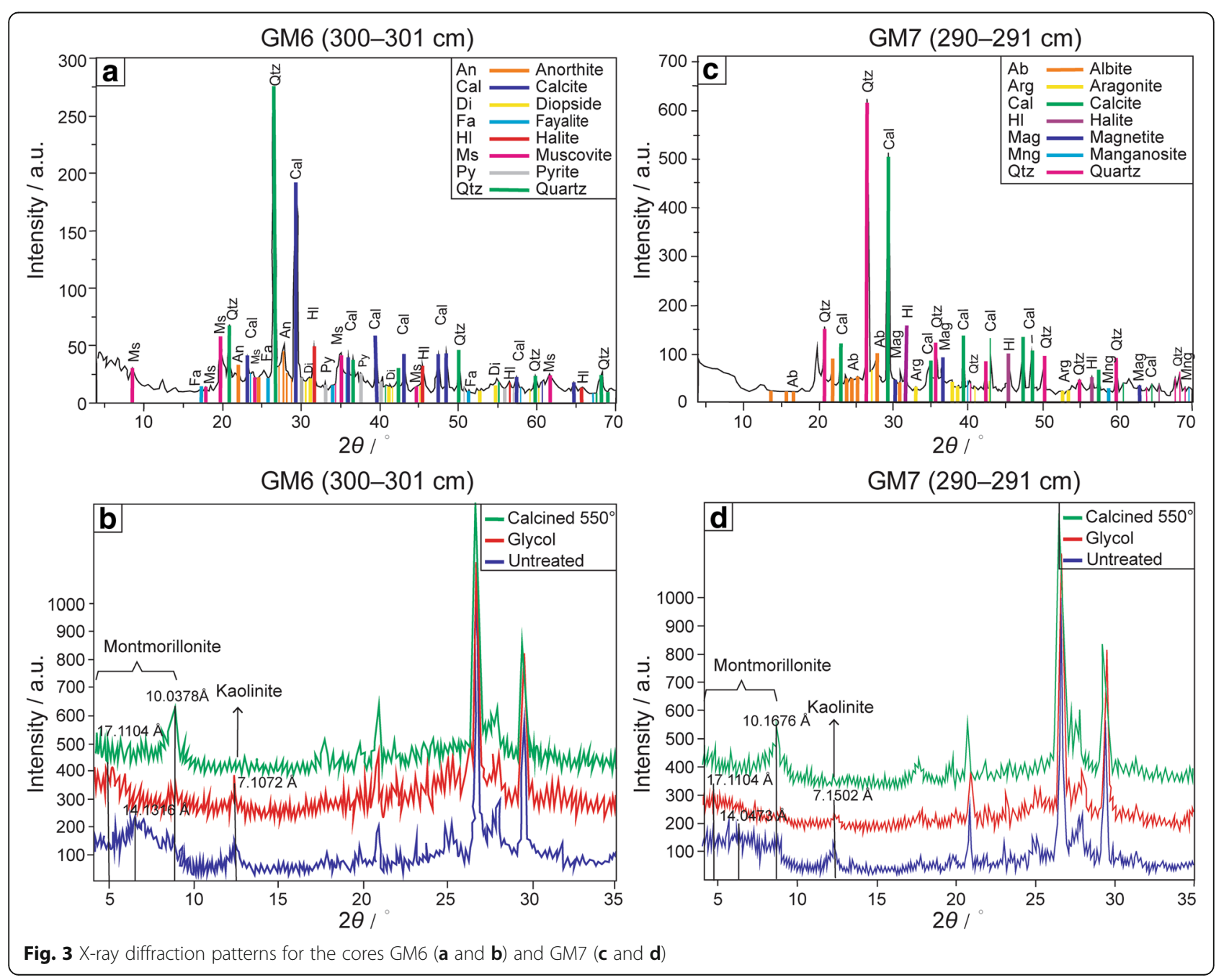



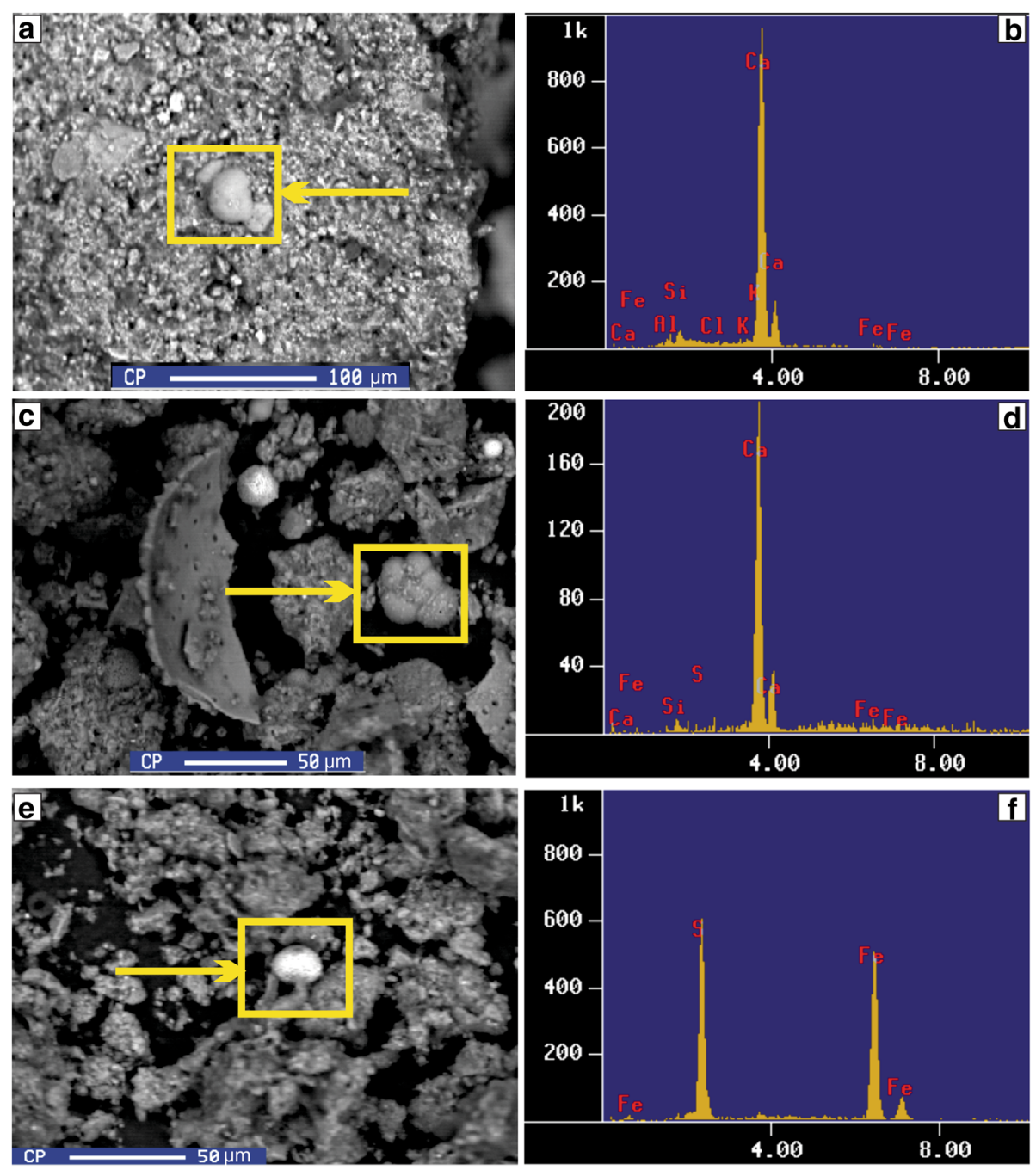

Fig. 4 SEM-EDS spectrum. The yellow rectangles in a, c, and e show the analysis positions. a, b Shell with calcite and plagioclase (GM6, 256-257 cm interval); c, d Foraminifer with calcite and halite (GM7, 300-301 cm interval); e, f Pyrite with sulfate and iron (GM6, 316-317 cm interval)

The enrichment of $\mathrm{MnO}$ is probably due to the mineral manganosite. The $\mathrm{Na}_{2} \mathrm{O}$ and $\mathrm{K}_{2} \mathrm{O}$ contents in the cores GM6 and GM7 are depleted relative to UCC, indicating that the sediments are low in feldspar content (Nagarajan et al. 2017). The enrichment of $\mathrm{Fe}_{2} \mathrm{O}_{3}$ and $\mathrm{TiO}_{2}$ contents is probably due to the presence of accessary minerals like magnetite and ilmenite, respectively (Papadopoulos et al. 2016). Geochemically, the sediments are classified mostly as shale (Fig. 6; Herron 1988).

\subsection{Trace element concentrations}

The trace element data for the GM6 and GM7 cores are listed in Table 4 and the UCC normalized patterns are illustrated in Fig. 7. The trace element contents between the GM6 and GM7 cores are almost similar, except Y and $\mathrm{Zn}$. The $\mathrm{Sc}, \mathrm{Cr}, \mathrm{Ni}$, and $\mathrm{Cu}$ contents in sediments are low relative to those in UCC. The low concentrations of $\mathrm{Rb}, \mathrm{Ba}$, and $\mathrm{U}$ in the core sediments reveal its mobility during sedimentary processes (Feng and Kerrich 1992). The $\mathrm{Zr}$ and Hf contents, which represent detrital input, are depleted in core sediments relative to those in UCC. The correlations between $\mathrm{Al}_{2} \mathrm{O}_{3}$ and $\mathrm{V}, \mathrm{Cr}$, and Sc for the GM6 ( $r=0.38,0.38$, and -0.19 , respectively; $n=$ 20. $r$ is correlation coefficient; the same follows) and GM7 $(r=0.39,0.34$, and 0.09 , respectively; $n=20)$ cores are not statistically significant, indicating that these elements are not associated with aluminosilicates (Tzifas et al. 2017; Zaid 2015; Zaid et al. 2017).

\subsection{Rare earth element concentrations}

The variation in $\Sigma$ REE contents in the cores GM6 (136$157 \mathrm{ppm})$ and GM7 (110-165 ppm) is statistically not significant (Table 5). The correlation between $\Sigma$ REE and 
Table 3 Major element concentrations (weight \%) for the deep sea sediments of the southwestern Gulf of Mexico

\begin{tabular}{|c|c|c|c|c|c|c|c|c|c|c|c|}
\hline Core No. & GM6 & & & & & & & & & & \\
\hline Section (cm) & $0-1$ & $31-32$ & $46-47$ & $61-62$ & $76-77$ & $91-92$ & $121-122$ & $136-137$ & 151-152 & $166-167$ & 180-181 \\
\hline vol. free $\mathrm{SiO}_{2}$ & 56.2 & 55.9 & 54.6 & 54.2 & 54.7 & 54.8 & 55.1 & 53.9 & 53.7 & 54.9 & 54.5 \\
\hline $\mathrm{SiO}_{2}$ & 45.7 & 45.3 & 44.1 & 44.1 & 45.5 & 45.2 & 44.8 & 44.1 & 43.8 & 46.0 & 44.7 \\
\hline $\mathrm{TiO}_{2}$ & 0.617 & 0.645 & 0.612 & 0.689 & 0.626 & 0.651 & 0.714 & 0.624 & 0.653 & 0.625 & 0.656 \\
\hline $\mathrm{Al}_{2} \mathrm{O}_{3}$ & 14.2 & 13.9 & 14.0 & 15.6 & 14.8 & 14.4 & 14.9 & 13.9 & 14.7 & 15.0 & 14.3 \\
\hline $\mathrm{Fe}_{2} \mathrm{O}_{3}{ }^{*}$ & 5.80 & 5.74 & 5.83 & 5.62 & 5.57 & 5.37 & 5.78 & 5.24 & 5.86 & 5.76 & 5.64 \\
\hline $\mathrm{MnO}$ & 0.048 & 0.054 & 0.127 & 0.041 & 0.050 & 0.053 & 0.081 & 0.095 & 0.075 & 0.086 & 0.680 \\
\hline $\mathrm{MgO}$ & 2.41 & 2.44 & 2.42 & 2.33 & 2.42 & 2.34 & 2.37 & 2.44 & 2.53 & 2.55 & 2.39 \\
\hline $\mathrm{CaO}$ & 7.68 & 7.98 & 8.92 & 8.36 & 9.48 & 10.2 & 8.46 & 11.2 & 9.54 & 8.94 & 9.33 \\
\hline $\mathrm{Na}_{2} \mathrm{O}$ & 3.49 & 3.54 & 3.42 & 3.27 & 2.61 & 2.36 & 2.65 & 2.25 & 2.68 & 2.60 & 2.57 \\
\hline $\mathrm{K}_{2} \mathrm{O}$ & 1.24 & 1.36 & 1.23 & 1.25 & 1.86 & 1.67 & 1.46 & 1.69 & 1.57 & 2.06 & 1.54 \\
\hline $\mathrm{P}_{2} \mathrm{O}_{5}$ & 0.119 & 0.125 & 0.121 & 0.136 & 0.222 & 0.231 & 0.124 & 0.239 & 0.204 & 0.212 & 0.216 \\
\hline LOI & 17.1 & 17.6 & 16.8 & 17.9 & 16.8 & 16.7 & 17.8 & 17.4 & 17.6 & 16.5 & 17.2 \\
\hline Sum & 98.3 & 98.5 & 97.5 & 99.3 & 99.9 & 99.1 & 99.0 & 99.1 & 99.1 & 100.3 & 99.1 \\
\hline $\mathrm{CIA}$ & 67 & 65 & 63 & 69 & 64 & 62 & 68 & 58 & 64 & 65 & 64 \\
\hline CIW & 72 & 70 & 67 & 74 & 69 & 67 & 73 & 63 & 69 & 72 & 69 \\
\hline PIA & 70 & 67 & 65 & 72 & 66 & 64 & 71 & 59 & 66 & 68 & 66 \\
\hline ICV & 1.50 & 1.57 & 1.61 & 1.38 & 1.53 & 1.57 & 1.45 & 1.70 & 1.56 & 1.51 & 1.60 \\
\hline $\mathrm{SiO}_{2} / \mathrm{Al}_{2} \mathrm{O}_{3}$ & 3.23 & 3.27 & 3.16 & 2.82 & 3.07 & 3.14 & 3.01 & 3.18 & 2.99 & 3.08 & 3.13 \\
\hline $\mathrm{K}_{2} \mathrm{O} / \mathrm{Na}_{2} \mathrm{O}$ & 0.354 & 0.383 & 0.359 & 0.382 & 0.711 & 0.708 & 0.549 & 0.748 & 0.587 & 0.795 & 0.598 \\
\hline $\mathrm{Al}_{2} \mathrm{O}_{3} / \mathrm{Na}_{2} \mathrm{O}$ & 4.05 & 3.91 & 4.09 & 4.79 & 5.67 & 6.09 & 5.62 & 6.15 & 5.47 & 5.76 & 5.54 \\
\hline $\mathrm{K}_{2} \mathrm{O} / \mathrm{Al}_{2} \mathrm{O}_{3}$ & 0.087 & 0.098 & 0.088 & 0.080 & 0.125 & 0.116 & 0.098 & 0.122 & 0.107 & 0.138 & 0.108 \\
\hline Section (cm) & 196-197 & $210-211$ & $222-223$ & $226-227$ & $241-242$ & $256-257$ & $300-301$ & $316-317$ & $330-331$ & \multicolumn{2}{|c|}{ Mean $\pm 1 s(n=20)$} \\
\hline vol. free $\mathrm{SiO}_{2}$ & 55.5 & 55.5 & 54.9 & 53.7 & 55.0 & 53.9 & 54.5 & 55.1 & 53.6 & \multicolumn{2}{|l|}{$54.7 \pm 0.74$} \\
\hline $\mathrm{SiO}_{2}$ & 45.5 & 44.8 & 44.9 & 44.9 & 45.2 & 44.0 & 44.7 & 45.8 & 44.4 & \multicolumn{2}{|l|}{$44.9 \pm 0.67$} \\
\hline $\mathrm{TiO}_{2}$ & 0.635 & 0.638 & 0.623 & 0.625 & 0.647 & 0.613 & 0.631 & 0.612 & 0.745 & \multicolumn{2}{|l|}{$0.64 \pm 0.04$} \\
\hline $\mathrm{Al}_{2} \mathrm{O}_{3}$ & 14.1 & 14.3 & 13.7 & 14.9 & 15.5 & 13.5 & 15.7 & 14.5 & 15.4 & \multicolumn{2}{|c|}{$14.6 \pm 0.66$} \\
\hline $\mathrm{Fe}_{2} \mathrm{O}_{3}{ }^{*}$ & 5.85 & 5.37 & 5.46 & 5.60 & 5.36 & 5.12 & 5.42 & 5.85 & 5.73 & \multicolumn{2}{|l|}{$5.6 \pm 0.23$} \\
\hline $\mathrm{MnO}$ & 0.785 & 0.096 & 0.076 & 1.835 & 0.086 & 0.139 & 0.074 & 0.470 & 0.650 & \multicolumn{2}{|l|}{$0.28 \pm 0.44$} \\
\hline $\mathrm{MgO}$ & 2.29 & 2.44 & 2.30 & 2.59 & 2.44 & 2.35 & 2.50 & 2.45 & 2.55 & \multicolumn{2}{|c|}{$2.43 \pm 0.09$} \\
\hline $\mathrm{CaO}$ & 8.25 & 8.79 & 10.8 & 8.39 & 8.76 & 11.7 & 8.86 & 9.06 & 8.97 & \multicolumn{2}{|c|}{$9.18 \pm 1.06$} \\
\hline $\mathrm{Na}_{2} \mathrm{O}$ & 2.72 & 2.25 & 2.34 & 2.72 & 2.34 & 2.56 & 2.21 & 2.55 & 2.21 & \multicolumn{2}{|c|}{$2.67 \pm 0.43$} \\
\hline $\mathrm{K}_{2} \mathrm{O}$ & 1.79 & 1.80 & 1.33 & 1.81 & 1.75 & 1.31 & 1.68 & 1.63 & 1.97 & \multicolumn{2}{|l|}{$1.60 \pm 0.25$} \\
\hline $\mathrm{P}_{2} \mathrm{O}_{5}$ & 0.120 & 0.229 & 0.234 & 0.192 & 0.236 & 0.251 & 0.224 & 0.201 & 0.216 & \multicolumn{2}{|c|}{$0.193 \pm 0.05$} \\
\hline LOI & 15.6 & 17.7 & 17.8 & 16.3 & 17.6 & 18.0 & 17.5 & 17.2 & 17.2 & \multicolumn{2}{|c|}{$17.2 \pm 0.61$} \\
\hline Sum & 97.6 & 98.4 & 99.5 & 100.0 & 99.9 & 99.6 & 99.5 & 100.3 & 100.0 & \multicolumn{2}{|l|}{$99.2 \pm 0.82$} \\
\hline $\mathrm{CIA}$ & 65 & 65 & 59 & 67 & 68 & 56 & 69 & 65 & 67 & \multicolumn{2}{|l|}{$65 \pm 4$} \\
\hline CIW & 71 & 71 & 63 & 73 & 74 & 60 & 75 & 70 & 74 & \multicolumn{2}{|l|}{$70 \pm 4$} \\
\hline PIA & 69 & 68 & 61 & 71 & 72 & 57 & 73 & 68 & 71 & \multicolumn{2}{|l|}{$67 \pm 4$} \\
\hline ICV & 1.59 & 1.50 & 1.67 & 1.58 & 1.38 & 1.76 & 1.36 & 1.56 & 1.48 & \multicolumn{2}{|c|}{$1.54 \pm 0.10$} \\
\hline $\mathrm{SiO}_{2} / \mathrm{Al}_{2} \mathrm{O}_{3}$ & 3.24 & 3.14 & 3.27 & 3.01 & 2.93 & 3.25 & 2.84 & 3.16 & 2.87 & \multicolumn{2}{|l|}{$3.09 \pm 0.14$} \\
\hline $\mathrm{K}_{2} \mathrm{O} / \mathrm{Na}_{2} \mathrm{O}$ & 0.659 & 0.800 & 0.568 & 0.665 & 0.748 & 0.512 & 0.760 & 0.639 & 0.888 & \multicolumn{2}{|c|}{$0.621 \pm 0.16$} \\
\hline $\mathrm{Al}_{2} \mathrm{O}_{3} / \mathrm{Na}_{2} \mathrm{O}$ & 5.18 & 6.35 & 5.87 & 5.50 & 6.60 & 5.28 & 7.12 & 5.70 & 6.97 & \multicolumn{2}{|c|}{$5.58 \pm 0.09$} \\
\hline $\mathrm{K}_{2} \mathrm{O} / \mathrm{Al}_{2} \mathrm{O}_{3}$ & 0.127 & 0.126 & 0.097 & 0.121 & 0.113 & 0.097 & 0.107 & 0.112 & 0.137 & $0.110+0.0$ & \\
\hline
\end{tabular}


Table 3 Major element concentrations (weight \%) for the deep sea sediments of the southwestern Gulf of Mexico (Continued)

\begin{tabular}{|c|c|c|c|c|c|c|c|c|c|c|c|}
\hline Core No. & GM7 & & & & & & & & & & \\
\hline Section (cm) & $0-1$ & $31-32$ & $46-47$ & $61-62$ & $76-77$ & $91-92$ & $121-122$ & $136-137$ & $151-152$ & 166-167 & 180-181 \\
\hline vol. free $\mathrm{SiO}_{2}$ & 54.6 & 55.0 & 54.0 & 55.0 & 54.8 & 54.1 & 53.5 & 54.9 & 56.5 & 54.4 & 56.0 \\
\hline $\mathrm{SiO}_{2}$ & 45.7 & 44.9 & 43.7 & 45.8 & 45.7 & 43.8 & 43.6 & 45.3 & 47.3 & 44.3 & 45.9 \\
\hline $\mathrm{TiO}_{2}$ & 0.605 & 0.670 & 0.570 & 0.680 & 0.604 & 0.571 & 0.585 & 0.621 & 0.680 & 0.618 & 0.740 \\
\hline $\mathrm{Al}_{2} \mathrm{O}_{3}$ & 15.0 & 15.9 & 13.7 & 15.7 & 14.4 & 13.2 & 13.4 & 14.0 & 14.8 & 14.4 & 14.7 \\
\hline $\mathrm{Fe}_{2} \mathrm{O}_{3}{ }^{*}$ & 5.80 & 5.13 & 4.96 & 4.98 & 5.82 & 4.95 & 4.90 & 5.26 & 5.12 & 5.45 & 5.78 \\
\hline $\mathrm{MnO}$ & 0.087 & 0.081 & 0.129 & 0.076 & 0.164 & 0.127 & 0.067 & 0.091 & 0.040 & 1.564 & 0.120 \\
\hline $\mathrm{MgO}$ & 2.46 & 2.56 & 2.35 & 2.68 & 2.49 & 2.33 & 2.24 & 2.36 & 2.31 & 2.53 & 1.98 \\
\hline $\mathrm{CaO}$ & 9.28 & 8.69 & 11.6 & 8.78 & 9.60 & 12.0 & 12.8 & 10.7 & 9.15 & 8.35 & 8.69 \\
\hline $\mathrm{Na}_{2} \mathrm{O}$ & 2.54 & 2.26 & 2.29 & 2.89 & 2.53 & 2.12 & 2.34 & 2.46 & 2.46 & 2.52 & 2.13 \\
\hline $\mathrm{K}_{2} \mathrm{O}$ & 2.06 & 1.26 & 1.47 & 1.48 & 1.90 & 1.63 & 1.37 & 1.40 & 1.58 & 1.44 & 1.79 \\
\hline $\mathrm{P}_{2} \mathrm{O}_{5}$ & 0.208 & 0.180 & 0.249 & 0.190 & 0.207 & 0.252 & 0.160 & 0.242 & 0.210 & 0.201 & 0.140 \\
\hline LOI & 16.0 & 18.0 & 19.0 & 16.7 & 17.2 & 18.5 & 18.5 & 16.9 & 16.2 & 17.5 & 17.6 \\
\hline Sum & 99.8 & 99.6 & 100.0 & 99.9 & 100.7 & 99.4 & 100.0 & 99.5 & 99.8 & 98.9 & 99.5 \\
\hline$C I A$ & 64 & 71 & 57 & 68 & 63 & 55 & 54 & 60 & 66 & 68 & 67 \\
\hline CIW & 71 & 76 & 61 & 73 & 68 & 59 & 57 & 64 & 71 & 72 & 73 \\
\hline PIA & 68 & 74 & 58 & 71 & 65 & 56 & 55 & 62 & 68 & 70 & 70 \\
\hline ICV & 1.52 & 1.30 & 1.71 & 1.38 & 1.60 & 1.80 & 1.80 & 1.63 & 1.44 & 1.56 & 1.45 \\
\hline $\mathrm{SiO}_{2} / \mathrm{Al}_{2} \mathrm{O}_{3}$ & 3.04 & 2.83 & 3.20 & 2.92 & 3.17 & 3.33 & 3.24 & 3.23 & 3.20 & 3.07 & 3.13 \\
\hline $\mathrm{K}_{2} \mathrm{O} / \mathrm{Na}_{2} \mathrm{O}$ & 0.812 & 0.558 & 0.643 & 0.512 & 0.752 & 0.766 & 0.585 & 0.570 & 0.642 & 0.573 & 0.840 \\
\hline $\mathrm{Al}_{2} \mathrm{O}_{3} / \mathrm{Na}_{2} \mathrm{O}$ & 5.92 & 7.02 & 5.98 & 5.43 & 5.70 & 6.20 & 5.74 & 5.71 & 6.01 & 5.72 & 6.88 \\
\hline $\mathrm{K}_{2} \mathrm{O} / \mathrm{Al}_{2} \mathrm{O}_{3}$ & 0.137 & 0.079 & 0.108 & 0.094 & 0.132 & 0.124 & 0.102 & 0.100 & 0.107 & 0.100 & 0.122 \\
\hline Section (cm) & 196-197 & $210-211$ & $222-223$ & $226-227$ & $241-242$ & $256-257$ & $300-301$ & $316-317$ & $330-331$ & \multicolumn{2}{|c|}{ Mean $\pm 1 s(n=20)$} \\
\hline vol. free $\mathrm{SiO}_{2}$ & 55.3 & 56.0 & 54.5 & 55.0 & 55.0 & 55.3 & 55.5 & 56.9 & 55.4 & \multicolumn{2}{|l|}{$55.1 \pm 0.83$} \\
\hline $\mathrm{SiO}_{2}$ & 46.7 & 47.0 & 44.3 & 45.0 & 44.7 & 45.3 & 45.4 & 47.7 & 45.2 & \multicolumn{2}{|l|}{$45.4 \pm 1.16$} \\
\hline $\mathrm{TiO}_{2}$ & 0.625 & 0.730 & 0.572 & 0.593 & 0.680 & 0.690 & 0.690 & 0.725 & 0.620 & \multicolumn{2}{|c|}{$0.643 \pm 0.056$} \\
\hline $\mathrm{Al}_{2} \mathrm{O}_{3}$ & 14.9 & 14.3 & 13.0 & 14.7 & 16.0 & 13.6 & 14.4 & 14.3 & 15.1 & \multicolumn{2}{|c|}{$14.5 \pm 0.84$} \\
\hline $\mathrm{Fe}_{2} \mathrm{O}_{3}{ }^{*}$ & 5.87 & 5.91 & 4.79 & 5.20 & 4.23 & 5.12 & 4.79 & 5.84 & 5.06 & \multicolumn{2}{|c|}{$5.25 \pm 0.46$} \\
\hline $\mathrm{MnO}$ & 0.114 & 0.250 & 0.114 & 0.081 & 0.180 & 0.078 & 0.260 & 0.061 & 0.080 & \multicolumn{2}{|c|}{$0.188 \pm 0.33$} \\
\hline $\mathrm{MgO}$ & 2.59 & 1.76 & 2.39 & 2.36 & 2.25 & 2.41 & 2.87 & 2.36 & 2.49 & \multicolumn{2}{|c|}{$2.39 \pm 0.24$} \\
\hline $\mathrm{CaO}$ & 8.53 & 9.46 & 12.3 & 8.69 & 8.36 & 9.56 & 8.49 & 8.26 & 8.37 & \multicolumn{2}{|c|}{$9.58 \pm 1.45$} \\
\hline $\mathrm{Na}_{2} \mathrm{O}$ & 2.82 & 2.24 & 2.14 & 2.76 & 2.36 & 2.65 & 2.68 & 2.73 & 2.49 & \multicolumn{2}{|c|}{$2.47 \pm 0.23$} \\
\hline $\mathrm{K}_{2} \mathrm{O}$ & 2.06 & 2.06 & 1.39 & 2.36 & 2.24 & 2.36 & 2.03 & 1.74 & 1.89 & \multicolumn{2}{|l|}{$1.78 \pm 0.35$} \\
\hline $\mathrm{P}_{2} \mathrm{O}_{5}$ & 0.188 & 0.230 & 0.262 & 0.109 & 0.270 & 0.117 & 0.190 & 0.188 & 0.170 & \multicolumn{2}{|c|}{$0.198 \pm 0.045$} \\
\hline LOI & 16.1 & 16.0 & 18.1 & 15.7 & 18.0 & 16.8 & 17.5 & 14.9 & 18.4 & \multicolumn{2}{|c|}{$17.2 \pm 1.11$} \\
\hline Sum & 100.5 & 99.9 & 99.3 & 97.6 & 99.2 & 98.6 & 99.2 & 98.8 & 99.8 & \multicolumn{2}{|l|}{$99.5 \pm 0.68$} \\
\hline$C I A$ & 66 & 63 & 54 & 64 & 69 & 59 & 65 & 66 & 68 & \multicolumn{2}{|l|}{$63 \pm 5$} \\
\hline CIW & 72 & 69 & 58 & 71 & 77 & 66 & 71 & 72 & 74 & \multicolumn{2}{|l|}{$69 \pm 6$} \\
\hline PIA & 69 & 66 & 55 & 68 & 74 & 61 & 68 & 69 & 72 & \multicolumn{2}{|l|}{$66 \pm 6$} \\
\hline ICV & 1.51 & 1.57 & 1.82 & 1.50 & 1.27 & 1.68 & 1.52 & 1.52 & 1.39 & \multicolumn{2}{|c|}{$1.55 \pm 0.16$} \\
\hline $\mathrm{SiO}_{2} / \mathrm{Al}_{2} \mathrm{O}_{3}$ & 3.13 & 3.29 & 3.40 & 3.06 & 2.80 & 3.33 & 3.15 & 3.34 & 2.99 & \multicolumn{2}{|l|}{$3.14 \pm 0.17$} \\
\hline $\mathrm{K}_{2} \mathrm{O} / \mathrm{Na}_{2} \mathrm{O}$ & 0.729 & 0.920 & 0.651 & 0.857 & 0.949 & 0.891 & 0.757 & 0.637 & 0.759 & \multicolumn{2}{|l|}{$0.72 \pm 0.13$} \\
\hline $\mathrm{Al}_{2} \mathrm{O}_{3} / \mathrm{Na}_{2} \mathrm{O}$ & 5.30 & 6.37 & 6.10 & 5.34 & 6.77 & 5.12 & 5.37 & 5.23 & 6.07 & \multicolumn{2}{|c|}{$5.90 \pm 0.55$} \\
\hline $\mathrm{K}_{2} \mathrm{O} / \mathrm{Al}_{2} \mathrm{O}_{3}$ & 0.138 & 0.144 & 0.107 & 0.160 & 0.140 & 0.174 & 0.141 & 0.122 & 0.125 & $0.123 \pm 0.1$ & \\
\hline
\end{tabular}




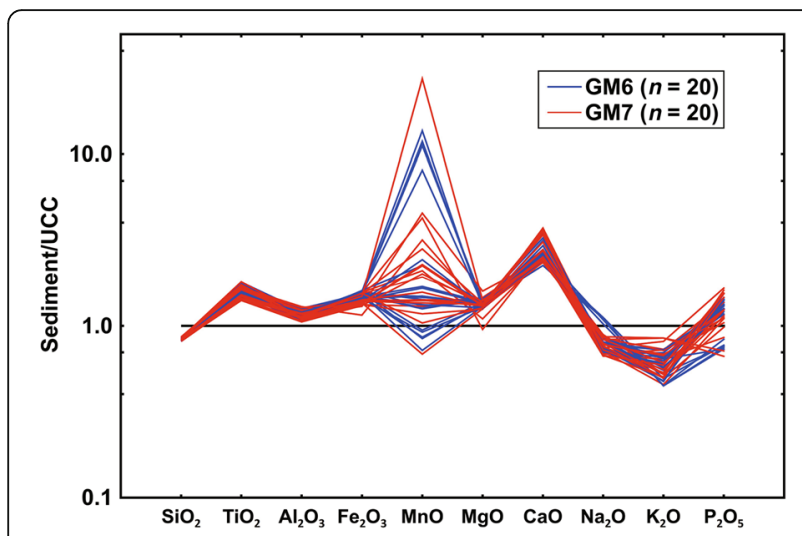

Fig. 5 Upper continental crust (UCC) normalized major element patterns (Taylor and McLennan 1985)

$\mathrm{Zr}$ contents is positive for the GM6 $(r=0.57, n=20)$ and GM7 ( $r=0.45, n=20)$ cores. On the other hand, the correlation between $\sum$ REE and $\mathrm{Al}_{2} \mathrm{O}_{3}$ is negative for the GM6 $(r=-0.14, n=20)$ and GM7 $(r=-0.15, n=20)$ cores, which indicates that the REE contents are housed in accessory mineral like zircon (Armstrong-Altrin et al. 2012; Periasamy and Venkateshwarlu 2017; Wang et al. 2017c, 2017d). The North American shale composite (NASC) normalized REE patterns for the GM6 and GM7 cores are characterized by flat light REE (LREE) and enriched heavy REE (HREE) with negative and positive $\mathrm{Eu}$ anomalies (Fig. 8). However, the $\mathrm{Eu}$ anomalies in cores GM6 (Eu/Eu" $=0.892$ to 1.03) and GM7 $\left(\mathrm{Eu} / \mathrm{Eu}^{*}=\right.$ 0.887 to 1.14 ) are similar (Table 5).

\section{Discussion}

\subsection{Palaeoweathering indices and sediment recycling}

The chemical composition of detrital sediments is highly useful to interpret the intensity of weathering as well as sediment recycling (Tawfik et al. 2017). Although huge

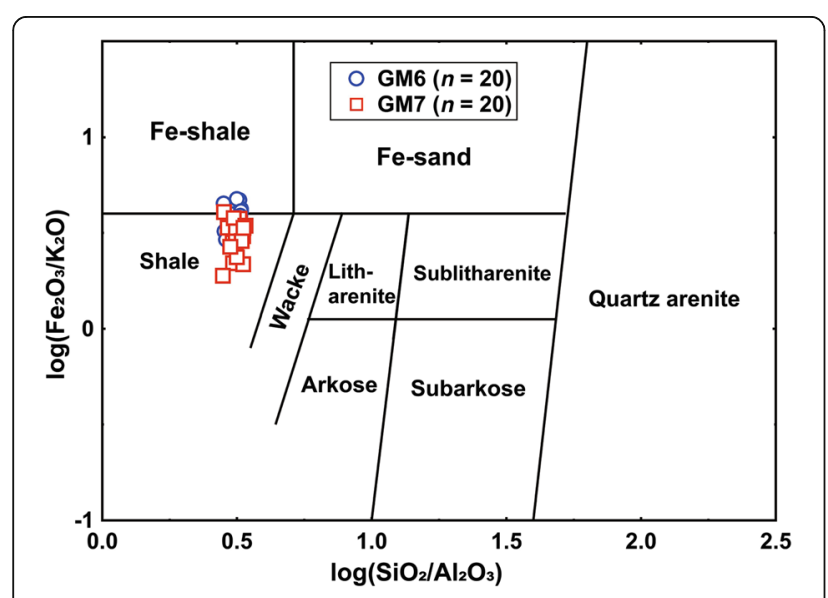

Fig. 6 Geochemical classification diagram for the core sediments, southwestern Gulf of Mexico (Herron 1988) number of weathering indices was proposed by various authors, in this study, we preferred the most commonly used weathering indices like CIA (Nesbitt and Young 1982), CIW (Harnois 1988), and PIA (Fedo et al. 1995). These weathering indices are represented by the following equations: $\mathrm{CIA}=\left[\mathrm{Al}_{2} \mathrm{O}_{3} /\left(\mathrm{Al}_{2} \mathrm{O}_{3}+\mathrm{CaO}^{*}+\mathrm{Na}_{2} \mathrm{O}+\right.\right.$ $\left.\left.\mathrm{K}_{2} \mathrm{O}\right)\right] \times 100, \mathrm{CIW}=\left[\mathrm{Al}_{2} \mathrm{O}_{3} / \mathrm{Al}_{2} \mathrm{O}_{3}+\mathrm{CaO}^{*}+\mathrm{Na}_{2} \mathrm{O}\right] \times 100$, and PIA $=\left[\mathrm{Al}_{2} \mathrm{O}_{3}-\mathrm{K}_{2} \mathrm{O} /\left(\mathrm{Al}_{2} \mathrm{O}_{3}+\mathrm{CaO}^{*}+\mathrm{Na}_{2} \mathrm{O}\right)\right] \times 100$, in which $\mathrm{CaO}^{*}$ represents $\mathrm{CaO}$ only incorporated in the silicate fraction. The $\mathrm{Ca}$ content in the silicate fraction was calculated by the equation: $\mathrm{Ca}=\mathrm{CaO}_{\mathrm{t}}-\mathrm{CaO}_{\text {trg }}$ and $\mathrm{CaO}_{\text {trg }}=\left(\mathrm{Al}_{2} \mathrm{O}_{3}\right)_{\mathrm{t}} \times\left(\mathrm{CaO} / \mathrm{Al}_{2} \mathrm{O}_{3}\right)_{\mathrm{UCC}}$, where " $\mathrm{t}$ " = the total abundance in the sample and "trg" = terrigenous.

The CIA, CIW, and PIA values increase if weathering increases and values of $\leq 50$ indicate low weathering (Fedo et al. 1995; Harnois 1988; Nesbitt and Young 1982). The CIA and CIW values for the GM6 core vary from 56 to 69 and from 60 to 75, respectively, and for GM7, which vary from 54 to 71 and from 57 to 77 , respectively (Table 3). Similarly, the PIA values for the core GM6 vary from 57 to 73 and for core GM7, which vary between 55 and 74 (Table 3). The CIA, CIW, and PIA values of the core sediments are indicating a moderate to intense weathering condition (Table 3). On the other hand, index of compositional variability $\left(\mathrm{ICV}=\left[\left(\mathrm{CaO}+\mathrm{K}_{2} \mathrm{O}+\mathrm{Na}_{2} \mathrm{O}+\mathrm{Fe}_{2} \mathrm{O}_{3}{ }^{(\mathrm{t})}+\right.\right.\right.$ $\left.\left.\mathrm{MgO}+\mathrm{MnO}+\mathrm{TiO}_{2}\right) / \mathrm{Al}_{2} \mathrm{O}_{3}\right] ;$ Cox et al. 1995), where $\mathrm{Fe}_{2} \mathrm{O}_{3}{ }^{(\mathrm{t})}$ represents total iron, is another method commonly used to infer sediment recycling, which decreases when weathering increases (Armstrong-Altrin et al. 2014, 2017; Madhavaraju et al. 2017; Nagarajan et al. 2017). According to Cox et al. (1995), the ICV value is high $(>1)$ in little-weathered detrital minerals like pyroxene and feldspar and it is low $(<1)$ in highly-weathered fine-grained sediments like clay. The ICV values for the core sediments GM6 (1.36-1.76) and GM7 (1.27-1.82) reveal a moderate weathering intensity in the source area (Table 3 ).

The elemental ratios such as $\mathrm{SiO}_{2} / \mathrm{Al}_{2} \mathrm{O}_{3}, \mathrm{Al}_{2} \mathrm{O}_{3} / \mathrm{Na}_{2} \mathrm{O}$, and $\mathrm{K}_{2} \mathrm{O} / \mathrm{Na}_{2} \mathrm{O}$ have been used extensively by various authors to interpret sediment recycling and are considered as a proxy to infer detrital input (Armstrong-Altrin 2009; Madhavaraju et al. 2016; Zaid 2013; Zaid et al. 2015). These ratios are $>6,>5$, and $>1$, respectively, if the sediment recycling is high (Tapia-Fernandez et al. 2017). The $\mathrm{SiO}_{2} / \mathrm{Al}_{2} \mathrm{O}_{3}, \mathrm{Al}_{2} \mathrm{O}_{3} / \mathrm{Na}_{2} \mathrm{O}$, and $\mathrm{K}_{2} \mathrm{O} / \mathrm{Na}_{2} \mathrm{O}$ ratios of the cores GM6 (2.82-3.27, 4-7, and 0.354-0.888, respectively) and GM7 (2.8-3.4, 5-7, and 0.512-0.949, respectively) are indicating low to moderate sediment recycling.

\subsection{Sediment provenance}

The provenance discrimination diagram of Roser and Korsch (1988) is widely preferred by various researchers to infer sediment provenance (Armstrong-Altrin 2009; Hernández-Hinojosa et al. 2018; Nagarajan et al. 2017; Tapia-Fernandez et al. 2017). The discrimination diagram 
Table 4 Trace element concentrations (ppm) for the deep sea sediments of the southwestern Gulf of Mexico

\begin{tabular}{|c|c|c|c|c|c|c|c|c|c|c|c|}
\hline Core No. & GM6 & & & & & & & & & & \\
\hline Section $(\mathrm{cm})$ & $0-1$ & $31-32$ & $46-47$ & $61-62$ & $76-77$ & $91-92$ & $121-122$ & $136-137$ & $151-152$ & $166-167$ & $180-181$ \\
\hline $\mathrm{Ba}$ & 378 & 425 & 339 & 385 & 301 & 360 & 365 & 369 & 435 & 380 & 465 \\
\hline Co & 26.2 & 25.5 & 22.1 & 23.7 & 18.9 & 23.6 & 24.8 & 21.9 & 19 & 22.8 & 21.7 \\
\hline $\mathrm{Cr}$ & 73.1 & 75.6 & 78.6 & 81.7 & 69.1 & 88.1 & 85.5 & 79.6 & 79.9 & 81.8 & 76.9 \\
\hline Cs & 7.4 & 5.64 & 7.89 & 5.78 & 7.31 & 7.93 & 6.54 & 7.22 & 4.89 & 8.32 & 4.79 \\
\hline $\mathrm{Cu}$ & 22.5 & 22.8 & 23 & 22.6 & 19.9 & 22.8 & 21.8 & 20.1 & 20.8 & 21.8 & 22.9 \\
\hline $\mathrm{Hf}$ & 3.31 & 3.21 & 3.45 & 3.45 & 2.99 & 3.64 & 2.98 & 3.42 & 3.16 & 3.21 & 3.07 \\
\hline Mo & 6.62 & 1.2 & 1.4 & 1.4 & 0.737 & 1 & 1.1 & 0.755 & 1.3 & 0.853 & 1.4 \\
\hline $\mathrm{Nb}$ & 12.5 & 10.5 & 13.5 & 11.8 & 11.8 & 13.6 & 12.6 & 13 & 12.5 & 13.4 & 12.7 \\
\hline $\mathrm{Ni}$ & 23 & 23.5 & 21.7 & 23.8 & 18 & 21.4 & 24.6 & 21.3 & 25 & 17.8 & 26 \\
\hline $\mathrm{Pb}$ & 24.8 & 15.9 & 24 & 17.9 & 16.2 & 15 & 16.4 & 14.1 & 17.9 & 16 & 14.8 \\
\hline $\mathrm{Rb}$ & 100 & 99.5 & 102.1 & 98.7 & 92.1 & 107 & 102.5 & 94 & 86.8 & 107.1 & 103.5 \\
\hline Sc & 14.1 & 12.7 & 15.3 & 14.6 & 12.9 & 15.3 & 13.8 & 14 & 12.9 & 14.8 & 10.8 \\
\hline $\mathrm{Sr}$ & 322.6 & 389.2 & 379.7 & 356.4 & 318.9 & 496.9 & 310.8 & 523.7 & 376.9 & 357.8 & 365.5 \\
\hline $\mathrm{Ta}$ & 0.449 & 0.436 & 0.497 & 0.48 & 0.45 & 0.401 & 0.86 & 0.497 & 1.25 & 0.447 & 0.75 \\
\hline Th & 10.1 & 9.87 & 10.4 & 8.65 & 9.66 & 10.2 & 6.78 & 10 & 7.45 & 10.3 & 8.65 \\
\hline U & 2.25 & 2.89 & 2.25 & 2.47 & 2.63 & 4.19 & 2.78 & 3.11 & 2.63 & 2.73 & 2.78 \\
\hline V & 116.6 & 118.4 & 119.4 & 112.8 & 115.1 & 118.9 & 135.6 & 107 & 117.7 & 124.7 & 129.5 \\
\hline Y & 22.2 & 25.6 & 24.1 & 9.85 & 21.1 & 25.4 & 19.6 & 24.2 & 18.7 & 24.2 & 8.39 \\
\hline $\mathrm{Zn}$ & 375.1 & 77.7 & 47.9 & 89.7 & 20 & 26 & 78.6 & 19 & 79.7 & 22 & 68.5 \\
\hline $\mathrm{Zr}$ & 119.2 & 88.7 & 133 & 79.7 & 109.4 & 138 & 96.3 & 127.7 & 112.4 & 126.8 & 102.5 \\
\hline $\mathrm{V} / \mathrm{Cr}$ & 1.60 & 1.57 & 1.52 & 1.38 & 1.67 & 1.35 & 1.59 & 1.34 & 1.47 & 1.52 & 1.68 \\
\hline $\mathrm{Ni} / \mathrm{Co}$ & 0.88 & 0.92 & 0.98 & 1.00 & 0.95 & 0.91 & 0.99 & 0.97 & 1.32 & 0.78 & 1.20 \\
\hline $\mathrm{Cu} / \mathrm{Zn}$ & 0.06 & 0.29 & 0.48 & 0.25 & 1.00 & 0.88 & 0.28 & 1.06 & 0.26 & 0.99 & 0.33 \\
\hline$U_{\mathrm{au}}$ & -1.10 & -0.40 & -1.21 & -0.41 & -0.59 & 0.80 & 0.52 & -0.23 & 0.15 & -0.69 & -0.10 \\
\hline Section $(\mathrm{cm})$ & 196-197 & $210-211$ & $222-223$ & $226-227$ & $241-242$ & $256-257$ & $300-301$ & $316-317$ & $330-331$ & \multicolumn{2}{|c|}{ Mean $\pm 1 s(n=20)$} \\
\hline $\mathrm{Ba}$ & 359 & 336 & 360 & 378 & 287 & 355 & 276 & 385 & 379 & \multicolumn{2}{|l|}{$366 \pm 46$} \\
\hline Co & 21.5 & 24.4 & 22.5 & 19.3 & 23.8 & 21.3 & 29 & 20.9 & 19.6 & \multicolumn{2}{|l|}{$22.6 \pm 2.59$} \\
\hline $\mathrm{Cr}$ & 67.6 & 84.5 & 77.5 & 62.8 & 94.8 & 70.7 & 92.7 & 71.8 & 84.7 & \multicolumn{2}{|l|}{$78.8 \pm 8.28$} \\
\hline Cs & 7.5 & 6.92 & 6.95 & 7.05 & 6.78 & 7.12 & 6.35 & 8.02 & 5.84 & \multicolumn{2}{|l|}{$6.81 \pm 1.00$} \\
\hline $\mathrm{Cu}$ & 21.1 & 20.9 & 21.4 & 18.2 & 23.7 & 19.9 & 19.9 & 21.6 & 22 & \multicolumn{2}{|l|}{$21.5 \pm 1.39$} \\
\hline $\mathrm{Hf}$ & 3.31 & 3.07 & 3.13 & 3.06 & 3.02 & 3.27 & 3.18 & 3.33 & 3.45 & \multicolumn{2}{|l|}{$3.24 \pm 0.18$} \\
\hline Mo & 4.51 & 0.727 & 0.91 & 0.261 & 1.3 & 0.712 & 1.4 & 1.52 & 1.8 & \multicolumn{2}{|l|}{$1.55 \pm 1.46$} \\
\hline $\mathrm{Nb}$ & 12.6 & 12.5 & 12.1 & 9 & 13.1 & 12.9 & 13.5 & 13.3 & 13.8 & \multicolumn{2}{|l|}{$12.5 \pm 1.13$} \\
\hline $\mathrm{Ni}$ & 21.5 & 26.3 & 27.9 & 13.6 & 27.9 & 18.8 & 28.5 & 16.2 & 28.8 & \multicolumn{2}{|l|}{$22.8 \pm 4.3$} \\
\hline $\mathrm{Pb}$ & 22.8 & 13.8 & 13.4 & 15 & 22.8 & 14 & 24.7 & 18.8 & 25.7 & \multicolumn{2}{|l|}{$18.2 \pm 4.26$} \\
\hline $\mathrm{Rb}$ & 95.4 & 93.6 & 95.3 & 88.4 & 101.5 & 96.4 & 99.9 & 106 & 102.7 & \multicolumn{2}{|l|}{$98.6 \pm 5.78$} \\
\hline Sc & 13.9 & 14.3 & 12.9 & 12.2 & 11.9 & 12.6 & 12.4 & 14.1 & 10.5 & \multicolumn{2}{|l|}{$13.3 \pm 1.35$} \\
\hline $\mathrm{Sr}$ & 330.2 & 525.1 & 463 & 311.5 & 366.8 & 534.5 & 325.5 & 359.6 & 371.9 & \multicolumn{2}{|l|}{$389 \pm 76$} \\
\hline $\mathrm{Ta}$ & 0.51 & 0.445 & 0.353 & 0.145 & 0.46 & 0.46 & 0.56 & 0.393 & 0.54 & \multicolumn{2}{|l|}{$0.52 \pm 0.22$} \\
\hline Th & 10.1 & 9.49 & 9.21 & 9.97 & 9.45 & 9.92 & 9.78 & 10.4 & 10.5 & \multicolumn{2}{|l|}{$9.54 \pm 0.98$} \\
\hline U & 2.05 & 3.04 & 3.09 & 1.95 & 3.12 & 2.61 & 3.14 & 2.09 & 3.25 & \multicolumn{2}{|l|}{$2.75 \pm 0.52$} \\
\hline V & 118.2 & 107.6 & 108.4 & 94.1 & 136.5 & 101.1 & 139.5 & 117.6 & 140.6 & \multicolumn{2}{|l|}{$119 \pm 13$} \\
\hline Y & 22.2 & 23.2 & 22.7 & 20.2 & 10.4 & 23.7 & 27.7 & 23.7 & 8.65 & \multicolumn{2}{|l|}{$20.3 \pm 6.02$} \\
\hline
\end{tabular}


Table 4 Trace element concentrations (ppm) for the deep sea sediments of the southwestern Gulf of Mexico (Continued)

\begin{tabular}{|c|c|c|c|c|c|c|c|c|c|c|c|}
\hline $\mathrm{Zn}$ & 51.1 & 38.9 & 25 & 20 & 43.3 & 23 & 60.4 & 26 & 126.5 & \multicolumn{2}{|c|}{$65.9 \pm 78.5$} \\
\hline $\mathrm{Zr}$ & 118.5 & 117.5 & 121.3 & 105.8 & 99.7 & 122.1 & 78.6 & 126.1 & 102.4 & \multicolumn{2}{|l|}{$111 \pm 17$} \\
\hline $\mathrm{V} / \mathrm{Cr}$ & 1.75 & 1.27 & 1.40 & 1.50 & 1.44 & 1.43 & 1.50 & 1.64 & 1.66 & \multicolumn{2}{|c|}{$1.51 \pm 0.13$} \\
\hline $\mathrm{Ni} / \mathrm{Co}$ & 1.00 & 1.08 & 1.24 & 0.70 & 1.17 & 0.88 & 0.98 & 0.78 & 1.47 & \multicolumn{2}{|c|}{$1.01 \pm 0.19$} \\
\hline $\mathrm{Cu} / \mathrm{Zn}$ & 0.41 & 0.54 & 0.86 & 0.91 & 0.55 & 0.87 & 0.33 & 0.83 & 0.17 & \multicolumn{2}{|c|}{$0.57 \pm 0.32$} \\
\hline$U_{\mathrm{au}}$ & -1.33 & -0.12 & 0.03 & -1.37 & -0.03 & -0.70 & -0.12 & -1.38 & -0.23 & \multicolumn{2}{|c|}{$-0.42 \pm 0.62$} \\
\hline Core No. & \multicolumn{11}{|l|}{ GM7 } \\
\hline Section $(\mathrm{cm})$ & $0-1$ & $31-32$ & $46-47$ & $61-62$ & $76-77$ & $91-92$ & $121-122$ & $136-137$ & $151-152$ & $166-167$ & $180-18$ \\
\hline $\mathrm{Ba}$ & 336 & 325 & 421 & 358 & 371 & 375 & 394 & 348 & 423 & 430 & 414 \\
\hline Co & 18.8 & 19.4 & 21.5 & 16.6 & 20.1 & 17.5 & 20.8 & 19.2 & 21.5 & 24.3 & 22.4 \\
\hline $\mathrm{Cr}$ & 65 & 75.4 & 66.9 & 78.5 & 70.3 & 42.8 & 69.8 & 59.8 & 79.8 & 69.4 & 82.6 \\
\hline Cs & 7.21 & 6.89 & 7.38 & 6.45 & 7.92 & 6.55 & 6.67 & 6.49 & 6.87 & 7.61 & 7.36 \\
\hline $\mathrm{Cu}$ & 18.7 & 19.6 & 20.1 & 20.5 & 20.5 & 17.8 & 20 & 18.3 & 23.5 & 21.6 & 19.7 \\
\hline $\mathrm{Hf}$ & 3.17 & 2.78 & 3.36 & 2.65 & 3.27 & 3.05 & 3.1 & 3.06 & 3.26 & 3.16 & 3.45 \\
\hline Mo & 0.736 & 0.756 & 0.746 & 0.814 & 0.864 & 0.752 & 0.745 & 0.71 & 0.478 & 0.267 & 0.642 \\
\hline $\mathrm{Nb}$ & 11.3 & 13.5 & 13.4 & 10.7 & 12.9 & 10.9 & 12.6 & 10.6 & 14.6 & 13.1 & 13.8 \\
\hline $\mathrm{Ni}$ & 13.6 & 33.6 & 18.4 & 28.6 & 15.5 & 19 & 21.3 & 15.9 & 34.2 & 15.8 & 31.6 \\
\hline $\mathrm{Pb}$ & 17.5 & 14.9 & 14.3 & 17 & 17.6 & 13.7 & 13.2 & 13.2 & 16.4 & 17.6 & 13.5 \\
\hline $\mathrm{Rb}$ & 93.7 & 95.7 & 97.7 & 78.6 & 104.2 & 88.1 & 90.6 & 87.9 & 81.7 & 99.7 & 79.4 \\
\hline SC & 12.3 & 11.7 & 13.2 & 12.5 & 13.6 & 11.6 & 13.2 & 11.2 & 13.7 & 13.8 & 11.5 \\
\hline $\mathrm{Sr}$ & 318.6 & 456.4 & 523.6 & 478.3 & 371.2 & 500.7 & 557.9 & 403.5 & 298.5 & 353.6 & 345.7 \\
\hline $\mathrm{Ta}$ & 0.405 & 1.25 & 0.509 & 0.69 & 0.419 & 0.374 & 0.465 & 0.324 & 0.56 & 0.433 & 0.48 \\
\hline Th & 10.1 & 11.7 & 10.6 & 9.58 & 10.5 & 9.86 & 9.5 & 9.02 & 9.68 & 9.88 & 9.48 \\
\hline U & 2.04 & 2.31 & 3.12 & 2.18 & 2.07 & 2.69 & 2.73 & 3.24 & 2.64 & 1.95 & 2.16 \\
\hline V & 103.9 & 125.4 & 104.8 & 140.3 & 118.1 & 84.8 & 99.1 & 95 & 98.6 & 112.7 & 175.6 \\
\hline Y & 20.6 & 28.7 & 23.4 & 23.5 & 23 & 21.1 & 22.6 & 20.3 & 21.5 & 22.9 & 20.7 \\
\hline $\mathrm{Zn}$ & 23.1 & 42.4 & 21 & 41.6 & 25 & 20 & 20 & 34.6 & 19.6 & 23 & 18.5 \\
\hline $\mathrm{Zr}$ & 105.3 & 110.6 & 124.3 & 14.8 & 120.3 & 107.6 & 118.5 & 108.9 & 99.6 & 121.4 & 125.6 \\
\hline $\mathrm{V} / \mathrm{Cr}$ & 1.60 & 1.66 & 1.57 & 1.79 & 1.68 & 1.98 & 1.42 & 1.59 & 1.24 & 1.62 & 2.13 \\
\hline $\mathrm{Ni} / \mathrm{Co}$ & 0.72 & 1.73 & 0.86 & 1.72 & 0.77 & 1.09 & 1.02 & 0.83 & 1.59 & 0.65 & 1.41 \\
\hline $\mathrm{Cu} / \mathrm{Zn}$ & 0.81 & 0.46 & 0.96 & 0.49 & 0.82 & 0.89 & 1.00 & 0.53 & 1.20 & 0.94 & 1.06 \\
\hline$U_{\mathrm{au}}$ & -1.31 & -1.57 & -0.41 & -1.01 & -1.43 & -0.60 & -0.43 & 0.23 & -0.59 & -1.34 & -1.00 \\
\hline Section $(\mathrm{cm})$ & 196-197 & $210-211$ & $222-223$ & $226-227$ & $241-242$ & $256-257$ & $300-301$ & $316-317$ & $330-331$ & Mean \pm 1 & $(n=20)$ \\
\hline $\mathrm{Ba}$ & 379 & 426 & 327 & 352 & 387 & 349 & 315 & 399 & 325 & $373 \pm 38$ & \\
\hline Co & 21.7 & 24.7 & 16 & 19.5 & 27.9 & 14.9 & 28.6 & 27.5 & 29.5 & $21.6 \pm 4.27$ & \\
\hline $\mathrm{Cr}$ & 77.3 & 74.7 & 57.5 & 69.6 & 68.6 & 51.3 & 71.9 & 108.6 & 75 & $70.7 \pm 13$. & \\
\hline Cs & 8.43 & 5.64 & 6.45 & 7.82 & 4.35 & 6.59 & 3.65 & 7.29 & 3.12 & $6.54 \pm 1.39$ & \\
\hline $\mathrm{Cu}$ & 22.2 & 19.8 & 16.9 & 21.4 & 21.6 & 20.2 & 22.3 & 24.3 & 21.5 & $20.5 \pm 1.85$ & \\
\hline $\mathrm{Hf}$ & 3.43 & 2.78 & 2.94 & 3.27 & 2.86 & 3.2 & 2.76 & 3.66 & 3.15 & $3.12 \pm 0.26$ & \\
\hline Mo & 0.888 & 0.812 & 0.679 & 0.969 & 0.843 & 1.24 & 0.972 & 0.973 & 0.856 & $1.21 \pm 1.0$ & \\
\hline $\mathrm{Nb}$ & 14.2 & 13.8 & 11.5 & 12.8 & 14.6 & 12.6 & 13.8 & 15.4 & 13.3 & $13.0 \pm 1.3$ & \\
\hline $\mathrm{Ni}$ & 17.1 & 29.8 & 24.3 & 21.6 & 35.4 & 20.1 & 39.8 & 29.5 & 32.1 & $24.8 \pm 7.99$ & \\
\hline $\mathrm{Pb}$ & 16.8 & 14.4 & 13.3 & 16.8 & 16.8 & 25.9 & 17.6 & 15.8 & 18.7 & $16.2 \pm 2.88$ & \\
\hline $\mathrm{Rb}$ & 107.2 & 83.6 & 88.8 & 100.9 & 92.5 & 90.5 & 98.6 & 100.6 & 97.7 & $93 \pm 8.2$ & \\
\hline Sc & 15.4 & 12.9 & 10.1 & 14.1 & 13.7 & 11.3 & 11.8 & 19.2 & 12.6 & $13 \pm 1.9$ & \\
\hline
\end{tabular}


Table 4 Trace element concentrations (ppm) for the deep sea sediments of the southwestern Gulf of Mexico (Continued)

\begin{tabular}{lllllllllll}
$\mathrm{Sr}$ & 347.4 & 412.4 & 561.4 & 335 & 432.5 & 350.6 & 416.4 & 459.1 & 468.4 & $420 \pm 79$ \\
$\mathrm{Ta}$ & 0.518 & 1.65 & 0.381 & 0.504 & 0.68 & 0.512 & 0.46 & 0.526 & 0.47 & $0.58 \pm 0.32$ \\
$\mathrm{Th}$ & 10.6 & 10.7 & 9.56 & 10.3 & 8.49 & 9.88 & 10.8 & 10.6 & 9.34 & $10 \pm 0.72$ \\
$\mathrm{U}$ & 2.44 & 2.34 & 2.75 & 2.95 & 2.17 & 2.67 & 2.62 & 3.07 & 2.49 & $2.53 \pm 0.38$ \\
$\mathrm{~V}$ & 122.3 & 123.5 & 87.5 & 116 & 118.6 & 101.6 & 137.6 & 153.6 & 103.5 & $116 \pm 23$ \\
$\mathrm{Y}$ & 24.8 & 21.8 & 21 & 22.7 & 21.6 & 22.9 & 22.8 & 26.7 & 23.9 & $22.8 \pm 2.07$ \\
$\mathrm{Zn}$ & 53 & 26.5 & 42.8 & 263.8 & 43.6 & 38.2 & 69.8 & 50.6 & 57.4 & $47 \pm 53$ \\
$\mathrm{Zr}$ & 133 & 134.3 & 101.2 & 118.4 & 89.5 & 101.8 & 110.5 & 152.8 & 114.3 & $111 \pm 27$ \\
$\mathrm{~V} / \mathrm{Cr}$ & 1.58 & 1.65 & 1.52 & 1.67 & 1.73 & 1.98 & 1.91 & 1.41 & 1.38 & $1.66 \pm 0.22$ \\
$\mathrm{Ni} / \mathrm{Co}$ & 0.79 & 1.21 & 1.52 & 1.11 & 1.27 & 1.35 & 1.39 & 1.07 & 1.09 & $1.16 \pm 0.33$ \\
$\mathrm{Cu} / \mathrm{Zn}$ & 0.42 & 0.75 & 0.39 & 0.08 & 0.50 & 0.53 & 0.32 & 0.48 & 0.37 & $0.65 \pm 0.30$ \\
$\mathrm{U}$ & -1.08 & -1.21 & -0.44 & -0.47 & -0.66 & -0.62 & -0.97 & -0.45 & -0.62 & $-0.80 \pm 0.44$ \\
\hline
\end{tabular}

Refer to Table 1, for water depth, latitude and longitude. Authigenic uranium $U_{a u}=$ Total $U-T h / 3$

of Roser and Korsch (1988) reveals that the sediments from the cores GM6 and GM7 were derived by the weathering of intermediate source rocks like basaltic andesite (Fig. 9). Similarly, the bivariate diagram based on La/Sc and $\mathrm{Co} / \mathrm{Th}$ ratios also suggests that the sediments were most probably derived from an intermediate source rock (Fig. 10).

The characteristics of the REE pattern and the Eu anomaly can be used as a tool to infer sediment provenance (Cullers et al. 1997). The REE patterns of the GM6 and GM7 core sediments are enriched in LREE and depleted in HREE with low negative to positive Eu anomalies, suggesting the derivation of sediments from an intermediate igneous rock. Furthermore, we compared the chondrite-normalized REE patterns of the core sediments with the potential source rocks from the southern Gulf of Mexico (Fig. 11). This comparison again demonstrates that the intermediate rocks as the probable source rocks for the two sediment cores, which is consistent with the geology of the Gulf of Mexico (Fig. 1).

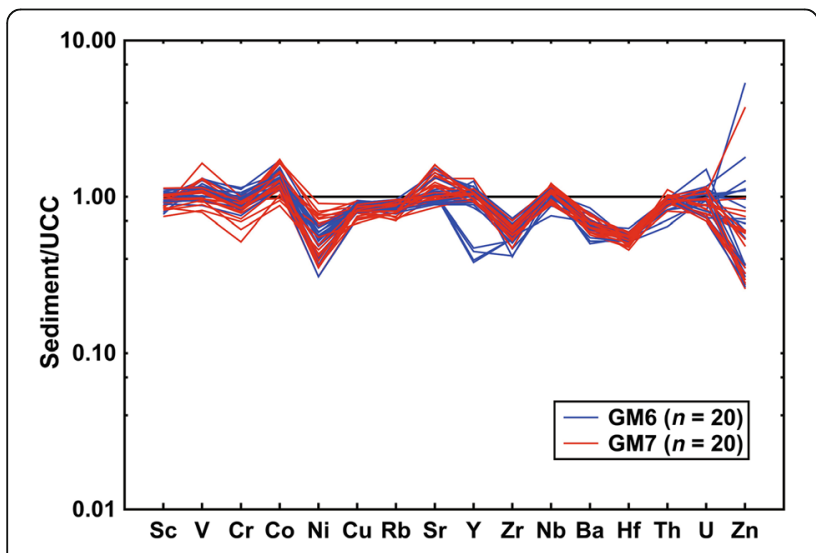

Fig. 7 Upper continental crust (UCC) normalized trace element patterns (Taylor and McLennan 1985)

\subsection{Redox-sensitive trace element concentrations}

Among other REEs, cerium (Ce) is sensitive to changes in redox conditions, so its depletion or enrichment can report changes in the oxygenation conditions of a depositional medium (Elderfield and Greaves 1982). A positive Ce anomaly $\left(\mathrm{Ce} / \mathrm{Ce}^{\prime \prime} \geq 1\right)$ in sediments suggests its deposition under an anoxic condition, whereas a negative $\mathrm{Ce}$ anomaly $(\mathrm{Ce} / \mathrm{Ce} "<1)$ represents an oxic condition (Tostevin et al. 2016). In this study, negative Ce anomaly $\left(\mathrm{Ce} / \mathrm{Ce}^{*}<1\right)$ is observed, which varies from 0.845 to 0.907 and from 0.84 to 0.986 for the GM6 and GM7 cores, respectively, indicating an oxic depositional condition (Table 5).

The $\mathrm{V} / \mathrm{Cr}$ ratio is considered as a good indicator of a redox condition, if this ratio is $>4.5$, which represents an anoxic condition, whereas $<2$ indicates an oxic condition in the depositional environment (Jones and Manning 1994). The V/Cr ratio in the GM6 (1.27-1.75) and GM7 (1.24 to 1.98 , except one sample with value 2.13 ) cores are $<2$ (Table 4), indicating an oxic condition. In addition, $\mathrm{Ni} / \mathrm{Co}$ ratio $<5$ and $>5$ indicate oxic and anoxic conditions, respectively (Jones and Manning 1994). The $\mathrm{Ni} / \mathrm{Co}$ ratios for the GM6 and GM7 cores range from 0.70 to 1.47 and from 0.65 to 1.73 , respectively, indicating an oxic condition. Similarly, the $\mathrm{Cu} / \mathrm{Zn}$ ratio is also a good paleo-redox indicator, which is always high $(>1)$ in the redox depositional condition (Goldberg and Humayun 2016). The $\mathrm{Cu} / \mathrm{Zn}$ ratios in the GM6 and GM7 core sediments are $<1(0.57 \pm 0.32$ and $0.65 \pm 0.30$, respectively), indicating an oxic condition. Wignall and Myers (1988) documented that the authigenic uranium $\left(U_{\mathrm{au}}=\right.$ Total $\left.\mathrm{U}-\mathrm{Th} / 3\right)$ values between 5 and 12 suggest an oxic depositional condition, whereas values $>12$ are indicative of suboxic and anoxic conditions. The authigenic uranium values in GM6 core range from - 1.38 to 0.620 and for the GM7 core, they vary from -1.57 to 0.231 , suggesting an oxic depositional condition. 
Table 5 Rare earth element concentrations (ppm) for the deep sea sediments of the Southwestern Gulf of Mexico

\begin{tabular}{|c|c|c|c|c|c|c|c|c|c|c|c|}
\hline Core No. & GM6 & & & & & & & & & & \\
\hline Section $(\mathrm{cm})$ & $0-1$ & $31-32$ & $46-47$ & $61-62$ & $76-77$ & $91-92$ & $121-122$ & 136-137 & $151-152$ & $166-167$ & 180-181 \\
\hline La & 30.3 & 28.7 & 32.9 & 30.5 & 29.5 & 33.4 & 31.3 & 32.3 & 30.7 & 33.4 & 29.8 \\
\hline $\mathrm{Ce}$ & 60.4 & 59.6 & 64.8 & 58.5 & 57.7 & 64.3 & 59.4 & 60.7 & 57.6 & 65 & 58.8 \\
\hline $\operatorname{Pr}$ & 7.3 & 7.15 & 7.95 & 7.26 & 7.11 & 8 & 7.45 & 7.58 & 6.74 & 7.93 & 6.89 \\
\hline $\mathrm{Nd}$ & 26.1 & 26.1 & 28.5 & 25.5 & 25.5 & 28.7 & 26.9 & 27.7 & 25.9 & 28.4 & 26.3 \\
\hline $\mathrm{Sm}$ & 5.31 & 5.26 & 5.71 & 5.25 & 5.02 & 5.68 & 5.14 & 5.48 & 5.25 & 5.52 & 5.24 \\
\hline $\mathrm{Eu}$ & 1.03 & 1.15 & 1.15 & 1.03 & 1.01 & 1.2 & 1.04 & 1.14 & 1.06 & 1.14 & 1.07 \\
\hline $\mathrm{Gd}$ & 4.49 & 4.52 & 4.83 & 4.79 & 4.32 & 4.95 & 4.65 & 4.86 & 4.24 & 4.88 & 4.58 \\
\hline $\mathrm{Tb}$ & 0.717 & 0.725 & 0.764 & 0.745 & 0.685 & 0.785 & 0.736 & 0.772 & 0.715 & 0.758 & 0.726 \\
\hline Dy & 3.96 & 3.95 & 4.2 & 3.9 & 3.79 & 4.28 & 3.77 & 4.08 & 3.77 & 4.12 & 3.78 \\
\hline $\mathrm{Ho}$ & 0.821 & 0.834 & 0.828 & 0.813 & 0.769 & 0.862 & 0.808 & 0.843 & 0.775 & 0.822 & 0.789 \\
\hline $\mathrm{Er}$ & 2.14 & 2.12 & 2.23 & 2.13 & 2.02 & 2.31 & 2.14 & 2.23 & 2.13 & 2.18 & 2.18 \\
\hline $\mathrm{Tm}$ & 0.31 & 0.311 & 0.334 & 0.301 & 0.305 & 0.325 & 0.291 & 0.33 & 0.295 & 0.316 & 0.308 \\
\hline $\mathrm{Yb}$ & 2 & 1.98 & 2.17 & 1.95 & 1.98 & 2.24 & 1.96 & 2.17 & 1.95 & 2.16 & 1.98 \\
\hline Lu & 0.303 & 0.301 & 0.324 & 0.302 & 0.294 & 0.32 & 0.291 & 0.32 & 0.29 & 0.324 & 0.31 \\
\hline LREE & 129 & 127 & 140 & 127 & 125 & 140 & 130 & 134 & 126 & 140 & 127 \\
\hline HREE & 14.7 & 14.7 & 15.7 & 14.9 & 14.2 & 16.1 & 14.6 & 15.6 & 14.2 & 15.6 & 14.7 \\
\hline$\sum$ REE & 145 & 143 & 157 & 143 & 140 & 157 & 146 & 151 & 141 & 157 & 143 \\
\hline $\mathrm{Eu} / \mathrm{Eu}^{*}$ & 0.93 & 1.03 & 0.958 & 0.898 & 0.952 & 0.991 & 0.934 & 0.967 & 0.982 & 0.968 & 0.959 \\
\hline $\mathrm{Ce} / \mathrm{Ce}^{*}$ & 0.885 & 0.907 & 0.873 & 0.855 & 0.868 & 0.856 & 0.848 & 0.845 & 0.871 & 0.87 & 0.893 \\
\hline Section $(\mathrm{cm})$ & 196-197 & $210-211$ & $222-223$ & $226-227$ & $241-242$ & $256-257$ & $300-301$ & $316-317$ & $330-331$ & \multicolumn{2}{|c|}{ Mean $\pm 1 \mathrm{~s}(n=20)$} \\
\hline La & 30.8 & 31.2 & 30.2 & 29 & 31.6 & 31.8 & 30.5 & 33.1 & 31.8 & \multicolumn{2}{|l|}{$31.1 \pm 1.40$} \\
\hline $\mathrm{Ce}$ & 60.4 & 59.1 & 57.3 & 55.1 & 60.5 & 60.1 & 60.2 & 65.2 & 60.5 & \multicolumn{2}{|l|}{$60.3 \pm 2.73$} \\
\hline $\operatorname{Pr}$ & 7.36 & 7.35 & 7.05 & 6.94 & 7.24 & 7.49 & 7.56 & 7.93 & 7.38 & \multicolumn{2}{|l|}{$7.38 \pm 0.36$} \\
\hline $\mathrm{Nd}$ & 26.3 & 26.7 & 25.4 & 24.8 & 26.6 & 26.8 & 27.5 & 28.2 & 27.5 & \multicolumn{2}{|l|}{$26.8 \pm 1.14$} \\
\hline $\mathrm{Sm}$ & 5.26 & 5.28 & 5.01 & 4.96 & 5.21 & 5.35 & 5.28 & 5.52 & 5.26 & \multicolumn{2}{|l|}{$5.30 \pm 0.20$} \\
\hline $\mathrm{Eu}$ & 1.06 & 1.08 & 1.06 & 0.98 & 0.96 & 1.13 & 1.06 & 1.1 & 1.04 & \multicolumn{2}{|l|}{$1.07 \pm 0.06$} \\
\hline $\mathrm{Gd}$ & 4.59 & 4.61 & 4.5 & 4.22 & 4.26 & 4.92 & 4.35 & 4.75 & 4.38 & \multicolumn{2}{|l|}{$4.58 \pm 0.24$} \\
\hline $\mathrm{Tb}$ & 0.733 & 0.718 & 0.705 & 0.67 & 0.715 & 0.75 & 0.715 & 0.752 & 0.735 & \multicolumn{2}{|l|}{$0.73 \pm 0.03$} \\
\hline Dy & 3.94 & 3.9 & 3.82 & 3.77 & 3.76 & 4.11 & 3.81 & 4.13 & 3.79 & \multicolumn{2}{|l|}{$3.93 \pm 0.17$} \\
\hline $\mathrm{Ho}$ & 0.811 & 0.796 & 0.782 & 0.747 & 0.836 & 0.828 & 0.802 & 0.835 & 0.815 & \multicolumn{2}{|l|}{$0.81 \pm 0.03$} \\
\hline $\mathrm{Er}$ & 2.12 & 2.1 & 2.06 & 2 & 2.11 & 2.14 & 2.12 & 2.28 & 2.09 & \multicolumn{2}{|l|}{$2.14 \pm 0.08$} \\
\hline $\mathrm{Tm}$ & 0.313 & 0.306 & 0.3 & 0.297 & 0.291 & 0.315 & 0.31 & 0.318 & 0.309 & \multicolumn{2}{|l|}{$0.31 \pm 0.01$} \\
\hline $\mathrm{Yb}$ & 2.1 & 2.02 & 1.99 & 1.98 & 2.01 & 2.15 & 2.01 & 2.15 & 2.03 & \multicolumn{2}{|l|}{$2.05 \pm 0.09$} \\
\hline $\mathrm{Lu}$ & 0.307 & 0.301 & 0.299 & 0.297 & 0.302 & 0.31 & 0.301 & 0.317 & 0.303 & \multicolumn{2}{|l|}{$0.31 \pm 0.01$} \\
\hline LREE & 130 & 130 & 125 & 121 & 131 & 132 & 131 & 140 & 132 & \multicolumn{2}{|l|}{$131 \pm 5.61$} \\
\hline HREE & 14.9 & 14.8 & 14.5 & 14.0 & 14.3 & 15.5 & 14.4 & 15.5 & 14.5 & \multicolumn{2}{|l|}{$14.9 \pm 0.60$} \\
\hline$\Sigma \mathrm{REE}$ & 146 & 145 & 140 & 136 & 146 & 148 & 146 & 157 & 148 & \multicolumn{2}{|l|}{$147 \pm 6.16$} \\
\hline $\mathrm{Eu} / \mathrm{Eu}^{*}$ & 0.945 & 0.962 & 0.98 & 0.935 & 0.892 & 0.968 & 0.974 & 0.942 & 0.945 & \multicolumn{2}{|c|}{$0.956 \pm 0.031$} \\
\hline $\mathrm{Ce} / \mathrm{Ce}^{*}$ & 0.875 & 0.85 & 0.855 & 0.845 & 0.869 & 0.848 & 0.865 & 0.877 & 0.86 & \multicolumn{2}{|c|}{$0.866 \pm 0.017$} \\
\hline Core No. & GM7 & & & & & & & & & & \\
\hline Section $(\mathrm{cm})$ & $0-1$ & $31-32$ & $46-47$ & $61-62$ & $76-77$ & $91-92$ & $121-122$ & $136-137$ & $151-152$ & $166-167$ & 180-181 \\
\hline La & 29.2 & 26.5 & 33.3 & 24.6 & 32.4 & 29.5 & 31.2 & 27.3 & 30.5 & 32 & 23.2 \\
\hline $\mathrm{Ce}$ & 57.1 & 53.3 & 63.3 & 50.2 & 63.4 & 55.3 & 59 & 52.3 & 61.9 & 62.3 & 49.8 \\
\hline
\end{tabular}


Table 5 Rare earth element concentrations (ppm) for the deep sea sediments of the Southwestern Gulf of Mexico (Continued)

\begin{tabular}{|c|c|c|c|c|c|c|c|c|c|c|c|}
\hline $\mathrm{Pr}$ & 6.96 & 6.28 & 7.85 & 5.76 & 7.72 & 6.87 & 7.28 & 6.43 & 7.13 & 7.55 & 4.72 \\
\hline $\mathrm{Nd}$ & 25.1 & 23.9 & 28.1 & 20.1 & 27.5 & 24.9 & 26 & 22.9 & 25.8 & 27.1 & 18.2 \\
\hline Sm & 5.02 & 4.22 & 5.46 & 3.98 & 5.37 & 4.91 & 5.06 & 4.62 & 5.45 & 5.29 & 3.37 \\
\hline $\mathrm{Eu}$ & 0.983 & 0.967 & 1.11 & 0.88 & 1.11 & 0.995 & 1.05 & 0.981 & 1.01 & 1.07 & 0.821 \\
\hline $\mathrm{Gd}$ & 4.26 & 4.02 & 4.8 & 3.68 & 4.64 & 4.39 & 4.57 & 4.18 & 4.6 & 4.65 & 3.13 \\
\hline $\mathrm{Tb}$ & 0.686 & 0.651 & 0.753 & 0.59 & 0.746 & 0.676 & 0.712 & 0.651 & 0.732 & 0.725 & 0.501 \\
\hline Dy & 3.83 & 3.52 & 3.99 & 2.84 & 4.17 & 3.75 & 3.84 & 3.66 & 3.95 & 3.9 & 2.92 \\
\hline Ho & 0.76 & 0.742 & 0.821 & 0.54 & 0.828 & 0.756 & 0.778 & 0.736 & 0.829 & 0.796 & 0.52 \\
\hline $\mathrm{Er}$ & 2.09 & 2.01 & 2.2 & 1.41 & 2.22 & 1.99 & 2.03 & 2 & 2.12 & 2.11 & 1.34 \\
\hline Tm & 0.302 & 0.273 & 0.318 & 0.193 & 0.327 & 0.294 & 0.307 & 0.287 & 0.309 & 0.309 & 0.171 \\
\hline $\mathrm{Yb}$ & 2.06 & 1.72 & 2.13 & 1.26 & 2.12 & 1.99 & 1.98 & 1.93 & 2.07 & 1.99 & 1.14 \\
\hline Lu & 0.295 & 0.253 & 0.317 & 0.184 & 0.319 & 0.287 & 0.291 & 0.275 & 0.325 & 0.299 & 0.161 \\
\hline LREE & 123 & 114 & 138 & 105 & 136 & 121 & 129 & 114 & 131 & 134 & 99 \\
\hline HREE & 14.3 & 13.2 & 15.3 & 10.7 & 15.4 & 14.1 & 14.5 & 13.7 & 14.9 & 14.8 & 9.9 \\
\hline$\sum$ REE & 139 & 128 & 154 & 116 & 153 & 137 & 144 & 128 & 147 & 150 & 110 \\
\hline $\mathrm{Eu} / \mathrm{Eu}^{*}$ & 0.933 & 1.03 & 0.952 & 1.01 & 0.976 & 0.941 & 0.963 & 0.98 & 0.887 & 0.944 & 1.11 \\
\hline $\mathrm{Ce} / \mathrm{Ce}^{*}$ & 0.872 & 0.9 & 0.852 & 0.92 & 0.872 & 0.845 & 0.853 & 0.859 & 0.914 & 0.874 & 0.93 \\
\hline Section (cm) & 196-197 & $210-211$ & $222-223$ & $226-227$ & $241-242$ & $256-257$ & $300-301$ & $316-317$ & $330-331$ & \multicolumn{2}{|c|}{ Mean $\pm 1 \mathrm{~s}(n=20)$} \\
\hline La & 34.3 & 31.4 & 29.2 & 31.7 & 34.2 & 30.9 & 24.9 & 35.1 & 28.3 & \multicolumn{2}{|c|}{$30 \pm 3.37$} \\
\hline $\mathrm{Ce}$ & 66.9 & 60.2 & 54.9 & 62.1 & 65.7 & 60.5 & 51.3 & 67.6 & 54.1 & \multicolumn{2}{|c|}{$58.6 \pm 5.64$} \\
\hline $\operatorname{Pr}$ & 8.18 & 6.41 & 6.93 & 7.62 & 7.92 & 6.94 & 5.12 & 8.35 & 6.81 & \multicolumn{2}{|c|}{$6.94 \pm 0.96$} \\
\hline $\mathrm{Nd}$ & 29.4 & 26.8 & 24.6 & 27.2 & 28.2 & 26.5 & 20.1 & 30.2 & 25.1 & \multicolumn{2}{|c|}{$25.4 \pm 3.13$} \\
\hline Sm & 5.71 & 4.48 & 4.91 & 5.3 & 5.21 & 5.14 & 3.46 & 5.93 & 4.25 & \multicolumn{2}{|c|}{$4.86 \pm 0.70$} \\
\hline Eu & 1.17 & 1.05 & 1 & 1.08 & 1.08 & 1.01 & 0.88 & 1.24 & 1.01 & \multicolumn{2}{|c|}{$1.03 \pm 0.10$} \\
\hline Gd & 4.89 & 4.17 & 4.32 & 4.59 & 4.82 & 4.39 & 3.34 & 5.13 & 3.93 & \multicolumn{2}{|c|}{$4.32 \pm 0.51$} \\
\hline $\mathrm{Tb}$ & 0.774 & 0.68 & 0.68 & 0.723 & 0.77 & 0.699 & 0.48 & 0.803 & 0.612 & \multicolumn{2}{|c|}{$0.682 \pm 0.085$} \\
\hline Dy & 4.23 & 3.78 & 3.77 & 4 & 4.32 & 3.94 & 2.39 & 4.37 & 3.32 & \multicolumn{2}{|c|}{$3.72 \pm 0.51$} \\
\hline Ho & 0.854 & 0.783 & 0.763 & 0.823 & 0.91 & 0.798 & 0.49 & 0.897 & 0.711 & \multicolumn{2}{|c|}{$0.757 \pm 0.115$} \\
\hline $\mathrm{Er}$ & 2.27 & 2.09 & 2.01 & 2.17 & 2.34 & 2.09 & 1.28 & 2.33 & 2.01 & \multicolumn{2}{|c|}{$2.01 \pm 0.305$} \\
\hline $\mathrm{Tm}$ & 0.343 & 0.289 & 0.296 & 0.314 & 0.351 & 0.309 & 0.181 & 0.339 & 0.31 & \multicolumn{2}{|c|}{$0.291 \pm 0.051$} \\
\hline $\mathrm{Yb}$ & 2.17 & 1.98 & 1.91 & 2.08 & 2.24 & 1.99 & 1.12 & 2.26 & 2.01 & \multicolumn{2}{|c|}{$1.91 \pm 0.34$} \\
\hline Lu & 0.332 & 0.271 & 0.285 & 0.307 & 0.361 & 0.298 & 0.171 & 0.346 & 0.291 & \multicolumn{2}{|c|}{$0.283 \pm 0.054$} \\
\hline LREE & 144 & 129 & 121 & 134 & 141 & 130 & 105 & 147 & 119 & \multicolumn{2}{|c|}{$126 \pm 14$} \\
\hline HREE & 15.9 & 14.0 & 14.0 & 15.0 & 16.1 & 14.5 & 9.5 & 16.5 & 13.2 & \multicolumn{2}{|c|}{$14 \pm 1.93$} \\
\hline$\sum$ REE & 161 & 144 & 136 & 150 & 158 & 146 & 115 & 165 & 133 & \multicolumn{2}{|c|}{$141 \pm 15$} \\
\hline $\mathrm{Eu} / \mathrm{Eu}^{*}$ & 0.97 & 1.07 & 0.956 & 0.966 & 0.946 & 0.937 & 1.14 & 0.985 & 1.09 & \multicolumn{2}{|c|}{$0.989 \pm 0.065$} \\
\hline $\mathrm{Ce} / \mathrm{Ce}^{*}$ & 0.869 & 0.921 & 0.84 & 0.87 & 0.87 & 0.898 & 0.986 & 0.859 & 0.848 & \multicolumn{2}{|c|}{$0.878 \pm 0.049$} \\
\hline
\end{tabular}

Refer to Table 1, for water depth, latitude and longitude. $\mathrm{Ce} / \mathrm{Ce}^{*}$ denotes $\mathrm{Ce}_{\text {NASC }} /\left[\left(\mathrm{La}_{\mathrm{NASC}}\right)\left(\mathrm{Pr}_{\mathrm{NASC}}\right)\right]^{1 / 2}, \mathrm{Eu} / \mathrm{Eu}^{*}=\mathrm{Eu}_{\text {NASC }} /\left[\left(\mathrm{Sm}_{\mathrm{NASC}}\right)\left(\mathrm{Gd}_{\text {NASC }}\right)\right]^{1 / 2}$. NASC $=$ North American shale composite (Gromet et al. 1984). LREE $=\mathrm{La}+\mathrm{Ce}+\mathrm{Pr}+\mathrm{Nd}+\mathrm{Sm} ; \mathrm{HREE}=\mathrm{Gd}+\mathrm{Tb}+\mathrm{Dy}+\mathrm{Ho}+\mathrm{Er}+\mathrm{Tm}+\mathrm{Yb}+\mathrm{Lu}$

\subsection{Distribution of benthic foraminifera}

Benthic foraminifers were analyzed throughout the two cores to look for evidence of oxygenation in sediments. Benthic foraminifers in low oxygen environments are characterized by small size and low diversity (typically 2 or 3 species make up to $80 \%$ of the population; Sen Gupta and Machain-Castillo 1993), since only a few species can adapt to such conditions and they proliferate in great numbers due to lack of competition in a food-rich environment. Therefore, its populations are very abundant (9,000-147,000 individual species per gram of sediment Ind/g; Perez-Cruz and Machain-Castillo 1990). The foraminiferal populations identified in both cores show moderate to high diversity (number of species $\mathrm{S}=36$ (GM6) 


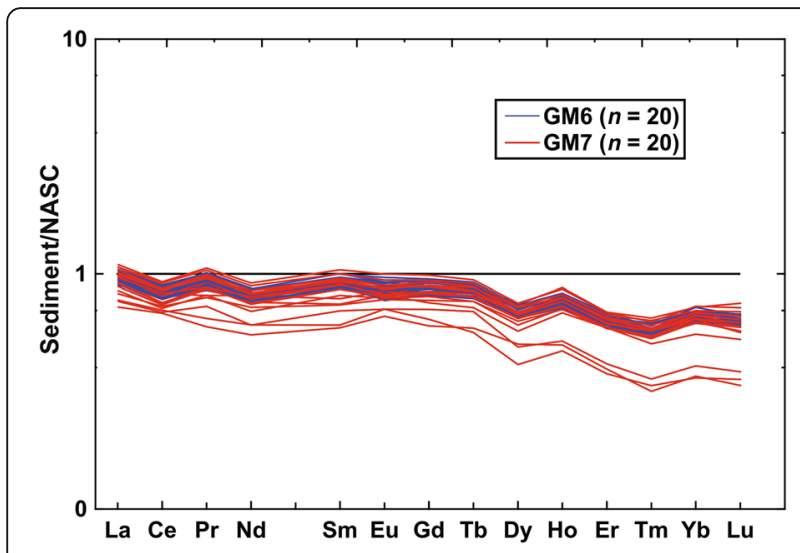

Fig. 8 North American shale composite (NASC) normalized REE patterns for the deep-sea sediments recovered from the southwestern Gulf of Mexico (Gromet et al. 1984)

and $\mathrm{S}=33(\mathrm{GM} 7))$ and abundances from 316 to 7840 Ind/g. Moreover, Alabaminella turgida, a species found to be sensitive to hypoxic conditions (Pflum and Frerichs 1976) is abundant throughout these cores, particularly during the Pleistocene. Another characteristic of severe hypoxia caused by enrichment of organic matter is the acidity of the water. Planktonic foraminifers dissolve easily in such environments (as the Mexican Pacific, Arellano-Torres et al. 2013) and are not well preserved or absent in the sediments beneath those environments. In the studied sediments, abundant and well preserved benthic and planktic foraminifers are found throughout the cores (Fig. 12). Similarly, our observations based on the distribution of benthic foraminifers are in agreement with the findings of Machain-Castillo et al. (1998) that

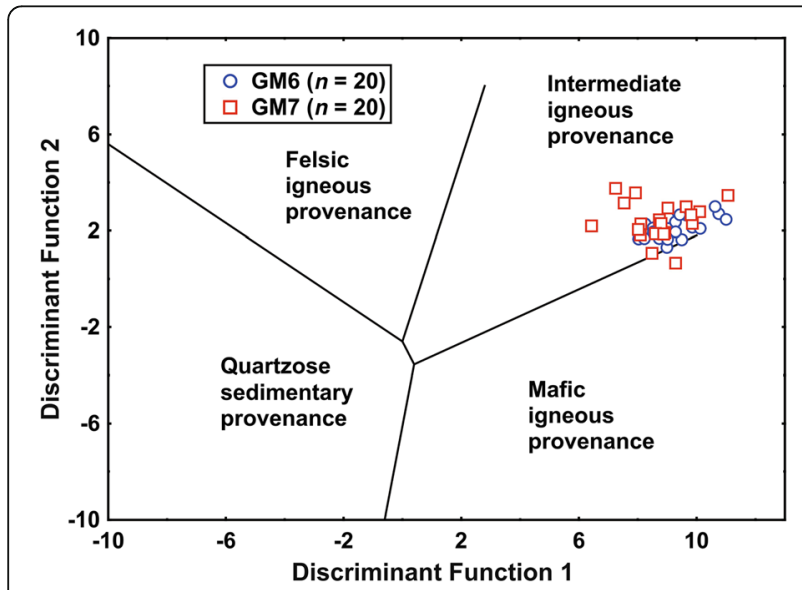

Fig. 9 Provenance discriminant function diagram of Roser and Korsch (1988). The discriminant functions are: Discriminant Function $1=\left(-1.773 \cdot \mathrm{TiO}_{2}\right)+\left(0.607 \cdot \mathrm{Al}_{2} \mathrm{O}_{3}\right)+\left(0.760 \cdot \mathrm{Fe}_{2} \mathrm{O}_{3}\right)+(-1.500 \cdot \mathrm{MgO})$ $+(0.616 \cdot \mathrm{CaO})+\left(0.509 \cdot \mathrm{Na}_{2} \mathrm{O}\right)+\left(-1.224 \cdot \mathrm{K}_{2} \mathrm{O}\right)+(-9.090)$; Discriminant Function $2=\left(0.445 \cdot \mathrm{TO}_{2}\right)+\left(0.070 \cdot \mathrm{Al}_{2} \mathrm{O}_{3}\right)+\left(-0.250 \cdot \mathrm{Fe}_{2} \mathrm{O}_{3}\right)+(-1.142 \cdot \mathrm{MgO})+(0.438 \cdot \mathrm{CaO})$ $+\left(1.475 \cdot \mathrm{Na}_{2} \mathrm{O}\right)+\left(1.426 \cdot \mathrm{K}_{2} \mathrm{O}\right) \mathrm{v}+(-6.861)$

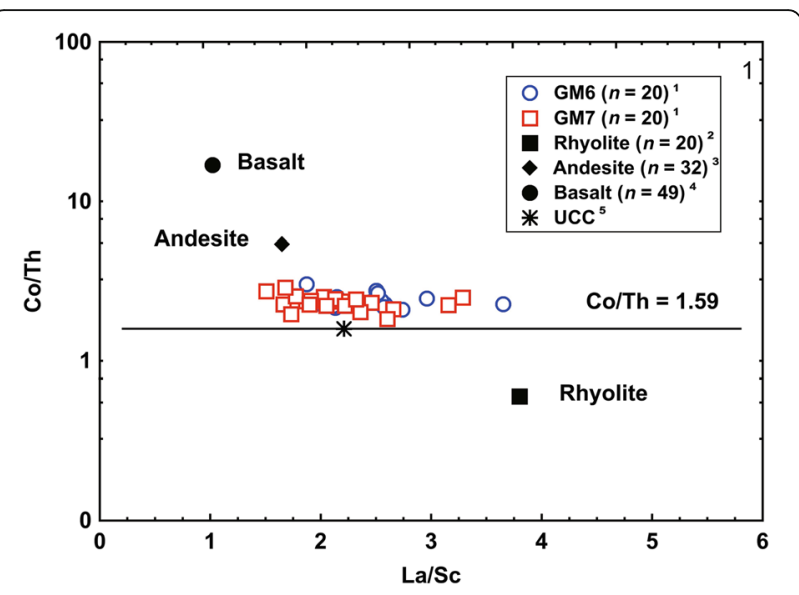

Fig. $10 \mathrm{La} / \mathrm{Sc}$ versus Co/Th bivariate plot for the deep-sea sediments recovered from the southwestern Gulf of Mexico. $n=$ number of samples; ${ }^{1}$ this study; ${ }^{2}$ Verma (2001), Nixon (1988); ${ }^{3}$ Orozco-Esquivel et al. (2007); ${ }^{4}$ Rodríguez et al. (2010), Schaaf et al. (2005); ${ }^{5}$ Taylor and McLennan (1985)

Pleistocene bathyal and abyssal waters were more oxygenated than present.

\subsection{Mineralogical characterization}

The low $\mathrm{K}_{2} \mathrm{O}$ and $\mathrm{Na}_{2} \mathrm{O}$ contents and $\mathrm{K}_{2} \mathrm{O} / \mathrm{Na}_{2} \mathrm{O}$ ratio suggests the low proportion of $\mathrm{K}$-feldspar and plagioclase in sediments (Dey et al. 2009; Nagarajan et al. 2007), which is also confirmed by XRD method in both sediment cores. The correlation between $\mathrm{SiO}_{2}$ versus $\mathrm{K}_{2} \mathrm{O}$ and $\mathrm{Na}_{2} \mathrm{O}$ in the cores GM6 $(r=-0.18$ and 0.39 , respectively, $n=20)$ and GM7 ( $r=0.27$ and 0.19 , respectively, $n=20$ ) are low, suggesting high mobility of $\mathrm{K}$ and $\mathrm{Na}$ at the time of weathering (Armstrong-Altrin and Machain-Castillo 2016; Tawfik et al. 2017). The $\mathrm{K}_{2} \mathrm{O} /$

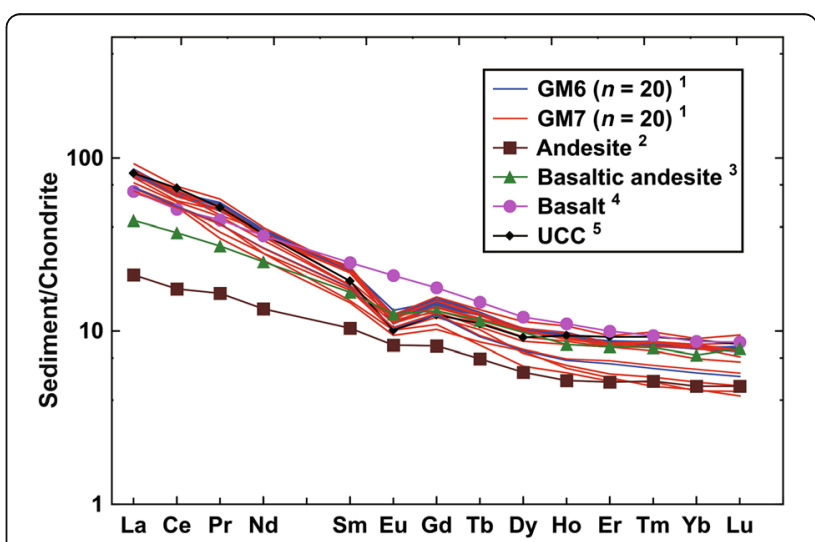

Fig. 11 Average chondrite-normalized rare earth element patterns for the deep-sea sediments. Normalization values are from Taylor and McLennan (1985), $n=$ number of samples; UCC = upper continental crust; ${ }^{1}$ this study; ${ }^{2}$ Verma (2001), Nixon $(1988) ;{ }^{3}$ Orozco-Esquivel et al. (2007); ${ }^{4}$ Rodríguez et al. (2010), Schaaf et al. (2005); ${ }^{5}$ Taylor and McLennan (1985) 

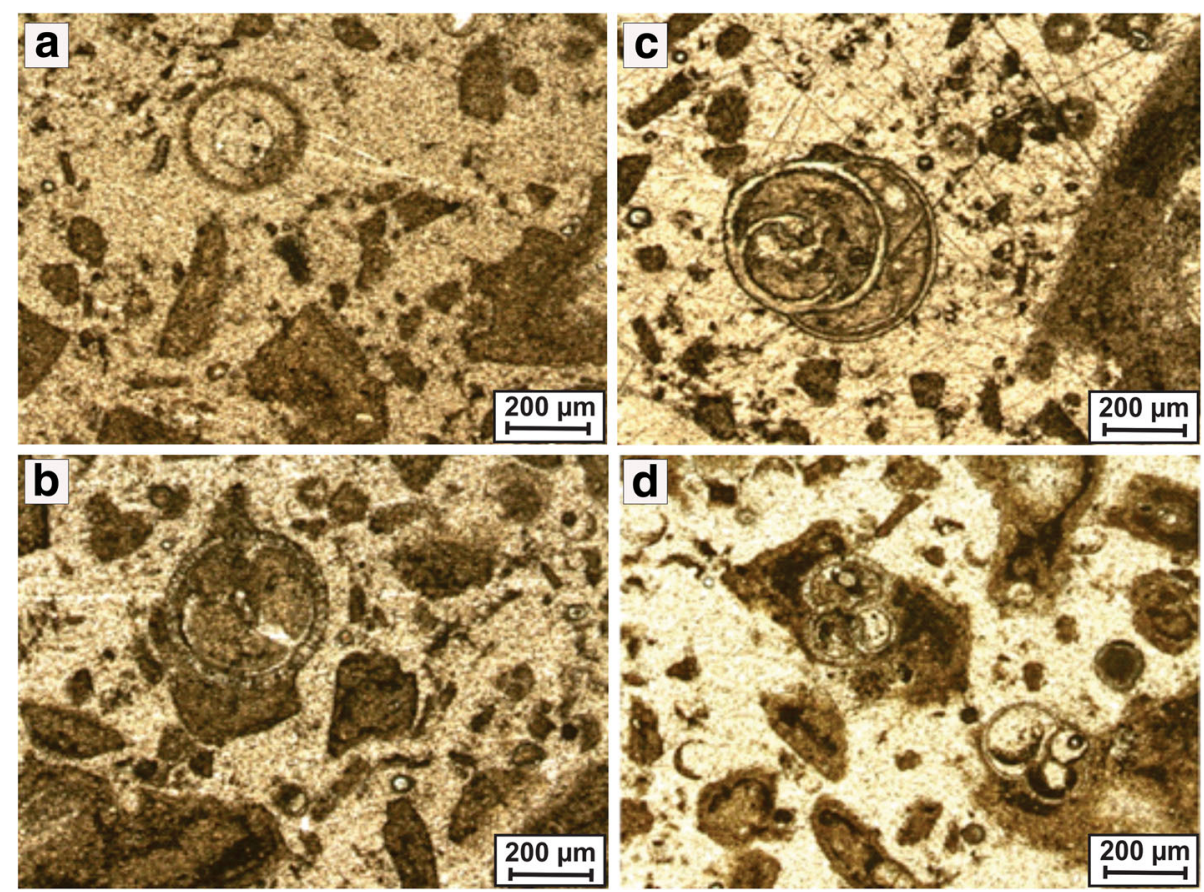

Fig. 12 Thin-section photomicrographs taken under plane-polarized light, showing biogenic components (planktonic foraminifers, globigerinids). a GM6 (31-32 cm interval); b GM6 (151-152 cm interval); c GM7 (31-32 cm interval); d GM7 (151-152 cm interval)

$\mathrm{Al}_{2} \mathrm{O}_{3}$ ratio in clastic sediments may indicate the abundance of alkali feldspars and aluminosilicates, which are $>5$ and $<5$, respectively (Cox et al. 1995). The low $\mathrm{K}_{2} \mathrm{O} /$ $\mathrm{Al}_{2} \mathrm{O}_{3}$ ratios in the GM6 (0.08-0.14) and GM7 (0.080.17 ) sediment cores indicate the higher abundance of aluminosilicates than alkali feldspars (Table 3).

The clay fraction of the GM6 and GM7 cores were dominated by kaolinite and smectite, which are related to the derivation of terrigenous materials from tropical regions (Abdullayev and Leroy 2016). Similarly, enrichment of $\mathrm{Cr}, \mathrm{Ga}, \mathrm{Ni}, \mathrm{Rb}, \mathrm{Y}$, and $\mathrm{Zn}$ concentrations in sediments is associated to the weathering of andesite (Saha et al. 2018). The correlations between $\mathrm{Al}_{2} \mathrm{O}_{3}$ versus $\mathrm{Nb}, \mathrm{Y}$, and $\mathrm{Zr}$ in the cores GM6 $(r=0.09,-0.47$, and -0.69 , respectively, $n=20)$ and GM7 $(r=0.26,0.04$, and -0.36 , respectively, $n=20$ ) are not statistically significant, suggesting that $\mathrm{Nb}, \mathrm{Y}$, and $\mathrm{Zr}$ are linked to the source rocks of intermediate composition and accessory detrital phases such as xenotime and zircon, respectively (Varghese et al. 2018). On the other hand, the enrichment of MnO content identified in the core sediments is most likely due to the concentration of manganosite mineral (Figs. 3c and 5). Numerous studies addressed the occurrence of manganosite in the Upper Jurassic carbonate platform shelf of the Molango Mn deposit, located near to the coastal region of the southwestern Gulf of Mexico (Johnson et al. 2016; Okita 1992; Okita and Shanks III 1992). Hence, we believe that manganosite in the core sediments was transported by the rivers to the deep-sea area of the Gulf of Mexico.
We consider that the enrichment of $\mathrm{CaO}$ in sediments is due to the distribution of sand-size benthic foraminifers, which is also identified at intervals of $256-257 \mathrm{~cm}$ (GM6) (Fig. 4a, b) and 300-301 cm (GM7) (Fig. 4c, d). In addition, the presence of calcite is related to the ability of smectite to observe cations such as $\mathrm{Ca}^{2+}$ to the surface of its crystalline structure (Abdullayev and Leroy 2016). Sulfate content identified in both cores by SEM-EDS represents the pyrite mineral (Fig. 4e, f). According to Berner (1984), pyrite is a widespread mineral and most marine sediments contain at least traces of it, since it forms during shallow burial by the reaction of detrital iron minerals with $\mathrm{H}_{2} \mathrm{~S}$ produced by bacterial sulfate reduction. The environment where this occurs is few centimeters below the water-surface interface. Most foraminifers live at the sediment water interface or just below it. Therefore, the foraminifers in the studied sediments were living under oxic conditions as revealed by their abundance, diversity and degree of preservation. Berner (1984) further indicated that in anoxic marine sediments, the high supply of organic matter and $\mathrm{H}_{2} \mathrm{~S}$ produce high concentrations of pyrite, which is not the case of the sediments studied, as seen in Figs. 3 and 4.

\section{Conclusions}

The compositional variations of two sediment cores recovered at the southwestern Gulf of Mexico were analyzed to infer their age and provenance. Texturally, the 
sediments are classified as silt and clay, and chemically as shale, indicating the domination of fine-grained sediments in both cores. Based on the radiocarbon ages the Pleistocene/Holocene boundary is identified at $130 \mathrm{~cm}$ interval. The weathering indices (CIA, CIW, and PIA) indicated a moderate to high intensity of weathering.

The concentrations of major element, transition trace elements, and the similarity between the chondrite- normalized REE patterns of the deep-sea sediments and the source rocks revealed that the depositional basin received sediments from the intermediate igneous rocks like andesite and basaltic andesite, at least during the last 23,615 cal yrs. BP.

The enrichment of $\mathrm{Zn}$ content in two intervals reveals an influence of anthropogenic input such as pesticides and agricultural waste in modern times. The authigenic uranium content, $\mathrm{Ce}$ anomaly, $\mathrm{V} / \mathrm{Cr}$ and $\mathrm{Ni} / \mathrm{Co}$ ratios and the distribution of benthic foraminifers suggested that the sediments were deposited in an oxic depositional condition. The similarity in geochemical composition between the two sediment cores reveals that the depositional condition in the deep-sea area of the southwestern Gulf of Mexico was similar at least in the last 23,615 cal yrs.

\section{Competing interests}

The authors declare that they have no competing interests.

\section{Authors' contributions}

AA carried out the XRD study and drafted the manuscript. JSA analyzed and interpreted the geochemistry data, and revised the manuscript for its suitability for publication. MLM interpreted the foraminiferal assemblages and carried out the ${ }^{14} \mathrm{C}$ dating study. PCM drafted the section "Study area". MAR participated in the XRD and SEM-EDS studies and drafted the section "Redox-sensitive trace element concentrations". All authors read and approved the final manuscript.

\section{Acknowledgements \\ John S. Armstrong-Altrin appreciates the partial financial assistance provided by the DGAPA-PAPIIT (No: IN106117) and the Institute of Marine Sciences and Limnology (ICML), UNAM, Internal (No. 616) projects. Abigail Anaya- Gregorio is thankful to DGAPA-PAPIIT, UNAM for the Bachelor degree schol- arship (No: IN106117). Mayla A. Ramos-Vázquez is grateful to the Posgrado en Ciencias del Mar y Limnologia (PCML) postgraduate program and to CONACYT for a doctoral fellowship (No. 595593/308610). \\ We extend our gratefulness to Eduardo Morales Garza and Ricardo M. for grain size analysis. We would like to thank Teodoro Hernández Treviño, Susana Santiago-Perez, Arturo Ronquillo A., and Hector M. Alexander Valdez for providing laboratory facilities. We thank the staffs Patricia Girón García, Carlos Linares-López, and Rufino Lozano Santacruz for their help in XRD, SEM-EDS, and XRF, respectively. We appreciate the support received by the Project "FACIES-PEMEX-PEP No: 420401851". We particularly thank the crew of Justo Sierra for their support during sampling. We would like to thank the Editors and four Reviewers for their constructive comments, which signifi- cantly improved our paper. Technical editing by Xiu-Fang Hu is highly appreciated.}

\section{Publisher's Note}

Springer Nature remains neutral with regard to jurisdictional claims in published maps and institutional affiliations.

\section{Author details}

'Licenciatura en Ingeniería en Geología Ambiental, Área Académica de Ciencias de la Tierra y Materiales, Universidad Autónoma del Estado de Hidalgo, Mineral de la Reforma, Hidalgo, Mexico. ${ }^{2}$ Instituto de Ciencias del Mar y Limnología, Universidad Nacional Autónoma de México, Unidad de Procesos Oceánicos y Costeros, Ciudad Universitaria, 04510 Ciudad de México, Mexico. ${ }^{3}$ Área Académica de Ciencias de la Tierra y Materiales, Universidad Autónoma del Estado de Hidalgo, Ciudad Universitaria, Carretera Pachuca-Tulancingo Km 4.5, Col. Carboneras, C.P. 42184 Mineral de la Reforma, Hidalgo, Mexico. ${ }^{4}$ Posgrado en Ciencias del Mar y Limnología, Universidad Nacional Autónoma de México, Ciudad Universitaria, 3000,

Coyoacán, C.P. 04510 Ciudad de México, Mexico.

Received: 23 January 2018 Accepted: 17 March 2018

Published online: 30 July 2018

\section{References}

Abdullayev, E., and S.A.G. Leroy. 2016. Provenance of clay minerals in the sediments from the Pliocene productive series, western South Caspian Basin Marine and Petroleum Geology 73: 517-527.

Arellano-Torres, E., M.L. Machain-Castillo, L.A. Contreras-Rosales, L.B. CuestaCastillo, and A.C. Ruiz-Fernández. 2013. Foraminiferal faunal evidence for glacial-interglacial variations in the ocean circulation and the upwelling of the Gulf of Tehuantepec (Mexico). Marine Micropaleontology 100: 52-66.

Armstrong-Altrin, J.S. 2009. Provenance of sands from Cazones, Acapulco, and Bahía Kino beaches, México. Revista Mexicana de Ciencias Geológicas 26 (3): 764-782.

Armstrong-Altrin, J.S. 2015. Evaluation of two multidimensional discrimination diagrams from beach and deep-sea sediments from the Gulf of Mexico and their application to Precambrian clastic sedimentary rocks. International Geology Review 57 (11-12): 1446-1461.

Armstrong-Altrin, J.S., Y.I. Lee, J.J. Kasper-Zubillaga, A. Carranza-Edwards, D. Garcia, G.N. Eby, V. Balaram, and N.L. Cruz-Ortiz. 2012. Geochemistry of beach sands along the western Gulf of Mexico, Mexico: Implication for provenance. Chemie der Erde - Geochemistry 72 (4): 345-362.

Armstrong-Altrin, J.S., Y.I. Lee, J.J. Kasper-Zubillaga, and E. Trejo-Ramírez. 2017. Mineralogy and geochemistry of sands along the Manzanillo and El Carrizal beach areas, southern Mexico: Implications for palaeoweathering, provenance and tectonic setting. Geological Journal 52 (4): 559-582.

Armstrong-Altrin, J.S., and M.L. Machain-Castillo. 2016. Mineralogy, geochemistry, and radiocarbon ages of deep sea sediments from the Gulf of Mexico, Mexico. Journal of South American Earth Sciences 71: 182-200.

Armstrong-Altrin, J.S., M.L. Machain-Castillo, L. Rosales-Hoz, A. Carranza-Edwards, J. Sanchez-Cabeza, and A.C. Ruíz-Fernández. 2015a. Provenance and depositional history of continental slope sediments in the southwestern Gulf of Mexico unraveled by geochemical analysis. Continental Shelf Research 95: 15-26.

Armstrong-Altrin, J.S., R. Nagarajan, V. Balaram, and O. Natalhy-Pineda. 2015b. Petrography and geochemistry of sands from the Chachalacas and Veracruz beach areas, western Gulf of Mexico, Mexico: Constraints on provenance and tectonic setting. Journal of South American Earth Sciences 64 (Part 1): 199-216.

Armstrong-Altrin, J.S., R. Nagarajan, Y.I. Lee, J.J. Kasper-Zubillaga, and L.P. Córdoba-Saldaña. 2014. Geochemistry of sands along the San Nicolás and San Carlos beaches, Gulf of California, Mexico: Implication for provenance. Turkish Journal of Earth Sciences 23 (5): 533-558.

Basu, A. 2017. Evolution of siliciclastic provenance inquiries: A critical appraisal. In Sediment provenance. Chapter 2, ed. R. Mazumder, 5-23. Netherlands: Elsevier Amsterdam. https://doi.org/10.1016/B978-0-12-803386-9.00002-2.

Berner, R.A. 1984. Sedimentary pyrite formation: An update. Geochimica et Cosmochimica Acta 48 (4): 605-615.

Bhattacharjee, J., K.K. Ghosh, and B. Bhattacharya. 2017. Petrography and geochemistry of sandstone-mudstone from Barakar formation (early Permian), Raniganj Basin, India: Implications for provenance, weathering and marine depositional conditions during lower Gondwana sedimentation. Geological Journal. https://doi.org/10.1002/gj.2946. in press.

Carranza-Edwards, A., E. Centeno-Garcia, L. Rosales-Hoz, and R. Lozano Santacruz. 2001. Provenance of beach gray sands from western México. Journal of South American Earth Sciences 14 (3): 291-305.

Cox, R., D.R. Lowe, and R.L. Cullers. 1995. The influence of sediment recycling and basement composition on evolution of mudrock chemistry in the southwestern United States. Geochimica et Cosmochimica Acta 59 (14): 2919-2940. 
Craigie, N. 2018. Production of chemostratigraphic correlation schemes. In Principles of elemental Chemostratigraphy. Advances in oil and gas exploration and production, 85-129. Cham: Springer.

Cullers, R.L., B. Bock, and C. Guidotti. 1997. Elemental distributions and neodymium isotopic compositions of Silurian metasediments, western Maine, USA: Redistribution of the rare earth elements. Geochimica et Cosmochimica Acta 61 (9): 1847-1861.

Day, J.W., A. Yañéz-Arancibia, W.J. Mitsch, A.L. Lara-Dominguez, J.N. Day, J.-Y. Ko, R. Lane, J. Lindsey, and D.Z. Lomeli. 2003. Using ecotechnology to address water quality and wetland habitat loss problems in the Mississippi Basin: A hierarchical approach. Biotechnology Advances 22 (1): 135-159.

Dey, S., A.K. Rai, and A. Chaki. 2009. Palaeoweathering, composition and tectonics of provenance of the Proterozoic intracratonic Kaladgi-Badami Basin, Karnataka, southern India: Evidence from sandstone petrography and geochemistry. Journal of Asian Earth Sciences 34 (6): 703-715.

Elderfield, H., and M.J. Greaves. 1982. The rare earth elements in seawater. Nature 296 (5854): 214-219.

Fedo, C.M., H.W. Nesbitt, and G.M. Young. 1995. Unraveling the effects of potassium metasomatism in sedimentary-rocks and paleosols, with implications for paleoweathering conditions and provenance. Geology 23 (10): 921-924.

Feng, R., and R. Kerrich. 1992. Geochemical evolution of granitoids from the Archean Abitibi southern volcanic zone and the Pontiac subprovince, Superior Province, Canada: Implications for tectonic history and source regions. Chemical Geology 98 (1-2): 23-70.

Folk, R.L. 1980. Petrology of sedimentary rocks, 182. Austin: Hemphill Publishing Company.

Goldberg, K., and M. Humayun. 2016. Geochemical paleoredox indicators in organic-rich shales of the Irati formation, Permian of the Paraná Basin, southern Brazil. Brazilian Journal of Geology 46 (3): 377-393.

Gromet, L.P., L.A. Haskin, R.L. Korotev, and R.F. Dymek. 1984. The "north American shale composite": Its compilation, major and trace element characteristics. Geochimica et Cosmochimica Acta 48 (12): 2469-2482.

Harnois, L. 1988. The CIW index: A new chemical index of weathering. Sedimentary Geology 55 (3-4): 319-322.

Hernández-Hinojosa, V., P.C. Montiel-García, J.S. Armstrong-Altrin, R. Nagarajan, and J.J. Kasper-Zubillaga. 2018. Textural and geochemical characteristics of beach sands along the western Gulf of Mexico, Mexico. Carpathian Journal of Earth and Environmental Sciences 13 (1): 161-174.

Herron, M.M. 1988. Geochemical classification of terrigenous sands and shales from core or log data. Journal of Sedimentary Research 58 (5): 820-829.

Hou, Q., C.L. Mou, Q.Y. Wang, and Z.Y. Tan. 2017. Provenance and tectonic setting of the early and middle Devonian Xueshan formation, the north Qilian Belt, China. Geological Journal. https://doi.org/10.1002/gj.2963. in press.

Jarvis, K.E. 1988. Inductively coupled plasma mass spectrometry: A new technique for the rapid or ultra-trace level determination of the rare-earth elements in geological materials. Chemical Geology 68 (1): 31-39.

Johnson, J.E., S.M. Webb, C. Ma, and W.W. Fischer. 2016. Manganese mineralogy and diagenesis in the sedimentary rock record. Geochimica et Cosmochimica Acta 173: 210-231.

Jones, B., and D.A.C. Manning. 1994. Comparison of geochemical indices used for the interpretation of palaeoredox conditions in ancient mudstones. Chemical Geology 111: 111-129.

Kasper-Zubillaga, J.J., J.S. Armstrong-Altrin, A. Carranza-Edwards, O. MortonBermea, and R. Lozano Santacruz. 2013. Control in beach and dune sands of the Gulf of Mexico and the role of nearby rivers. International Journal of Geosciences 4 (8): 1157-1174.

Kennett, J.P., K. Elmstrom, and N. Penrose. 1985. The last deglaciation in Orca Basin, Gulf of Mexico: High-resolution planktonic foraminiferal changes. Palaeogeography, Palaeoclimatology, Palaeoecology 50 (2-3): 189-216.

Kennett, J.P., and P. Huddlestun. 1972. Late Pleistocene paleoclimatology, foraminiferal biostratigraphy and tephrochronology, western Gulf of Mexico. Quaternary Research 2 (1): 38-69.

Lozano, R., and J.P. Bernal. 2005. Characterization of a new set of eight geochemical reference materials for XRF major and trace element analysis. Revista Mexicana de Ciencias Geológicas 22: 329-344.

Machain-Castillo, M.-L., B.K. Sen Gupta, and J.A. Alcala Herrera. 1998. Late quaternary change in deep-bathyal and abyssal waters of the Gulf of Mexico: Preservation record of the foraminifer Biloculinella irregularis. Journal of Foraminiferal Research 28 (2): 95-101.

Madhavaraju, J., S.A. Pacheco-Olivas, C.M. González-León, I.G. EspinozaMaldonado, P.A. Sanchez-Medrano, U. Villanueva-Amadoz, R. Monreal, T. Pi-
Puig, E. Ramírez-Montoya, and F.J. Grijalva-Noriega. 2017. Mineralogy and geochemistry of the lower cretaceous siliciclastic rocks of the Morita formation, sierra San José section, Sonora, Mexico. Journal of South American Earth Sciences 76: 397-411.

Madhavaraju, J., M. Tom, Y.I. Lee, V. Balaram, S. Ramasamy, A. Carranza-Edwards, and A. Ramachandran. 2016. Provenance and tectonic settings of sands from Puerto Peñasco, Desemboque and Bahia Kino beaches, gulf of California, Sonora, México. Journal of South American Earth Sciences 71: 262-275.

Mitra, R., G. Chakrabarti, and D. Shome. 2017. Geochemistry of the PalaeoMesoproterozoic Tadpatri shales, Cuddapah Basin, India: Implications on provenance, paleoweathering and paleoredox conditions. Acta Geochimica. https://doi.org/10.1007/s11631-017-0254-3. in press.

Nagarajan, R., J.S. Armstrong-Altrin, F.L. Kessler, and J. Jong. 2017. Petrological and geochemical constraints on provenance, paleo-weathering and tectonic setting of clastic sediments from the Neogene Lambir and Sibuti Formations, northwest Borneo. In Sediment provenance. Chapter 7, ed. R. Mazumder, 123153. Netherlands: Elsevier Amsterdam.

Nagarajan, R., J. Madhavaraju, R. Nagendra, J.S. Armstrong-Altrin, and J. Moutte. 2007. Geochemistry of Neoproterozoic shales of the Rabanpalli formation, Bhima Basin, northern Karnataka, southern India: Implications for provenance and paleoredox conditions. Revista Mexicana de Ciencias Geológicas 24 (2): 150-160.

Nesbitt, H.W., and G.M. Young. 1982. Early Proterozoic climates and plate motions inferred from major element chemistry of lutites. Nature 299 (5885): 715-717.

Nixon, G.T. 1988. Petrology of the younger andesites and dacites of Iztaccíhuatl volcano, Mexico: II. Chemical stratigraphy, magma mixing, and the composition of basaltic magma influx. Journal of Petrology 29 (2): 265-303.

Okita, P.M. 1992. Manganese carbonate mineralization in the Molango District, Mexico. Economic Geology 87 (5): 1345-1366.

Okita, P.M., and W.C. Shanks III. 1992. Origin of stratiform sediment-hosted manganese carbonate ore deposits: Examples from Molango, Mexico, and TaoJiang, China. Chemical Geology 99 (1-3): 139-163.

Orozco-Esquivel, T., C.M. Petrone, L. Ferrari, T. Tagami, and P. Manetti. 2007. Geochemical and isotopic variability in lavas from the eastern trans-Mexican Volcanic Belt: Slab detachment in a subduction zone with varying dip. Lithos 93 (1-2): 149-174.

Paikaray, S., S. Banerjee, and S. Mukherji. 2008. Geochemistry of shales from the Paleoproterozoic to Neoproterozoic Vindhyan Supergroup: Implications on provenance, tectonics and paleoweathering. Journal of Asian Earth Sciences 32 (1): 34-48.

Papadopoulos, A., A. Koroneos, G. Christofides, L. Papadopoulou, I. Tzifas, and S. Stoulos. 2016. Assessment of gamma radiation exposure of beach sands in highly touristic areas associated with plutonic rocks of the Atticocycladic zone (Greece). Journal of Environmental Radioactivity 162-163: 235-243.

Perez-Cruz, L.L., and M.L. Machain-Castillo. 1990. Benthic foraminifera of the oxygen minimum zone, continental shelf of the Gulf of Tehuantepec, Mexico. Journal of Foraminiferal Research 20 (4): 312-325.

Periasamy, V., and M. Venkateshwarlu. 2017. Petrography and geochemistry of Jurassic sandstones from the Jhuran formation of Jara dome, Kachchh Basin, India: Implications for provenance and tectonic setting. Journal of Earth System Science 126 (4): 44.

Pflum, C.E., and W.E. Frerichs. 1976. Gulf of Mexico deep-water foraminifers. Cushman Foundation for Foraminiferal Research Special Publication 14: 1-125.

Ramos-Vázquez, M.A., J.S. Armstrong-Altrin, L. Rosales-Hoz, M.L. Machain-Castillo, and A. Carranza-Edwards. 2017. Geochemistry of deep-sea sediments in two cores retrieved at the mouth of the Coatzacoalcos River delta, western Gulf of Mexico, Mexico. Arabian Journal of Geosciences 10 (6): 148.

Rodríguez, S.R., W. Morales-Barrera, P. Layer, and E. González-Mercado. 2010. A quaternary monogenetic volcanic field in the Xalapa region, eastern transMexican Volcanic Belt: Geology, distribution and morphology of the volcanic vents. Journal of Volcanology and Geothermal Research 197 (1): 149-166.

Rosales-Hoz, L., A. Carranza-Edwards, R.G. Martínez-Serrano, M.A. Alatorre, and J.S. Armstrong-Altrin. 2015. Textural and geochemical characteristics of marine sediments in the SW Gulf of Mexico: Implications for source and seasonal change. Environmental Monitoring Assessment 187 (4): 205.

Roser, B.P., and R.J. Korsch. 1988. Provenance signatures of sandstone-mudstone suites determined using discriminant function analysis of major-element data. Chemical Geology 67 (1-2): 119-139.

Saha, S., S. Banerjee, S.D. Burley, A. Ghosh, and P.K. Saraswati. 2010. The influence of flood basaltic source terrains on the efficiency of tectonic setting discrimination diagrams: An example from the Gulf of Khambhat, western India. Sedimentary Geology 228 (1-2): 1-13. 
Saha, S., S. Burley, and S. Banerjee. 2018. Mixing processes in modern estuarine sediments from the Gulf of Khambhat, western India. Marine and Petroleum Geology 91: 599-621.

Salas-de-León, D.A., M.A. Monreal-Gómez, M.A. Díaz-Flores, D. Salas-Monreal, H. Velasco-Mendoza, M.L. Riverón-Enzástiga, and G. Ortiz-Zamora. 2008. Role of near-bottom currents in the distribution of sediments within the Southern Bay of Campeche, Gulf of Mexico. Journal of Coastal Research 24 (6): 1487-1494.

Schaaf, P., J. Stimac, C. Siebe, and J.L. Macías. 2005. Geochemical evidence for mantle origin and crustal processes in volcanic rocks from Popocatépetl and surrounding monogenetic volcanoes, Central Mexico. Journal of Petrology 46 (6): $1243-1282$.

Selvaraj, K., T.Y. Lee, J.Y.T. Yang, E.A. Canuel, J.C. Huang, M. Dai, J.T. Liu, and S.J. Kao. 2015. Stable isotopic and biomarker evidence of terrigenous organic matter export to the deep sea during tropical storms. Marine Geology 364: 32-42.

Sen Gupta, B.K., and M.L. Machain-Castillo. 1993. Benthic foraminifera in oxygenpoor habitats. Marine Micropaleontology 20 (3-4): 183-201.

Solari, L.A., R. Torres de León, G. Hernández Pineda, J. Solé, G. Solís-Pichardo, and T. Henández-Treviño. 2007. Tectonic significance of cretaceous-tertiary magmatic and structural evolution of the northern margin of the Xolapa complex, Tierra Colorada area, southern Mexico. GSA Bulletin 119 (9): 1265-1279.

Stuiver, M., and P.J. Reimer. 1993. Extended ${ }^{14} \mathrm{C}$ database and revised CALIB radiocarbon calibration program. Radiocarbon 35 (1): 215-230.

Tapia-Fernandez, H.J., J.S. Armstrong-Altrin, and K. Selvaraj. 2017. Geochemistry and $\mathrm{U}-\mathrm{Pb}$ geochronology of detrital zircons in the Brujas beach sands, Campeche, southwestern Gulf of Mexico, Mexico. Journal of South American Earth Sciences 76: 346-361.

Tawfik, H.A., M.K. Salah, W. Maejima, J.S. Armstrong-Altrin, A.-M.T. Abdel-Hameed, M.M. El Ghandour, and A. Ruffell. 2017. Petrography and geochemistry of the lower Miocene Moghra sandstones, Qattara depression, north Western Desert, Egypt. Geological Journal. https://doi.org/10.1002/gj.3025. in press.

Taylor, S.R., and S.M. McLennan. 1985. The continental crust: Its composition and evolution. London: Blackwell Scientific Publications.

Tostevin, R., G.A. Shields, G.M. Tarbuck, T. He, M.O. Clarkson, and R.A. Wood. 2016. Effective use of cerium anomalies as a redox proxy in carbonate-dominated marine settings. Chemical Geology 438: 146-162.

Tzifas, I.T. P. Misaelides, A. Godelitsas, P.N. Gamaletsos, P. Nomikou, A.G. Karydas, V. Kantarelou, and A. Papadopoulos. 2017. Geochemistry of coastal sands of eastern Mediterranean: The case of Nisyros volcanic materials. Chemie der Erde - Geochemistry 77: 487-501.

Varghese, T.I., P.T. Nageshrao, N. Raghavendramurthy, and N. Ramasamy. 2018. Sediment geochemistry of coastal environments, southern Kerala, India: Implication for provenance. Arabian Journal of Geosciences 11 (3): 61.

Verma, S.P. 2001. Geochemical evidence for a rift-related origin of bimodal volcanism at Meseta Rio San Juan, north-central Mexican volcanic belt International Geology Review 43 (6): 475-493.

Verma, S.P., and J.S. Armstrong-Altrin. 2016. Geochemical discrimination of siliciclastic sediments from active and passive margin settings. Sedimentary Geology 332 (1): 1-12

Verma, S.P., L. Díaz-González, and J.S. Armstrong-Altrin. 2016a. Application of a new computer program for tectonic discrimination of Cambrian to Holocene clastic sediments. Earth Science Informatics 9 (2): 151-165.

Verma, S.P., D. Torres-Sánchez, F. Velasco-Tapia, K.S.V. Subramanyam, C. Manikyamba, and R. Bhutani. 2016b. Geochemistry and petrogenesis of extension-related magmas close to the volcanic front of the central part of the trans-Mexican Volcanic Belt. Journal of South American Earth Sciences 72: 126-136.

Wang, Z.W., X.G. Fu, X.L. Feng, C.Y. Song, D. Wang, W.B. Chen, and S.Q. Zeng. 2017a. Geochemical features of the black shales from the Wuyu Basin, southern Tibet: Implications for palaeoenvironment and palaeoclimate. Geological Journal 52 (2): 282-297

Wang, Z.W., J. Wang, X.G. Fu, X.L. Feng, D. Wang, C.Y. Song, W.B. Chen, and S.Q. Zeng. 2017c. Petrography and geochemistry of upper Triassic sandstones from the Tumengela formation in the Woruo Mountain area, north Qiangtang Basin, Tibet: Implications for provenance, source area weathering, and tectonic setting. Island Arc 26 (4): e12191.

Wang, Z.W., J. Wang, X.G. Fu, X.L. Feng, D. Wang, C.Y. Song, W.B. Chen, S.Q. Zeng, and F. Yu. 2017d. Provenance and tectonic setting of the Quemoco sandstones in the north Qiangtang Basin, North Tibet: Evidence from geochemistry and detrital zircon geochronology. Geological Journal. https:// doi.org/10.1002/gj.2967. in press.
Wang, Z.W., J. Wang, X.G. Fu, W.Z. Zhan, F. Yu, X.L. Feng, C.Y. Song, W.B. Chen, and S.Q. Zeng. 2017b. Organic material accumulation of Carnian mudstones in the north Qiangtang depression, eastern Tethys: Controlled by the paleoclimate, paleoenvironment, and provenance. Marine and Petroleum Geology 88: 440-457.

Wignall, P.B., and K.J. Myers. 1988. Interpreting benthic oxygen levels in mudrocks: A new approach. Geology 16 (5): 452-455.

Zaid, S.M. 2013. Provenance, diagenesis, tectonic setting and reservoir quality of the sandstones of the Kareem Formation, Gulf of Suez, Egypt. Journal of African Earth Sciences 85: 31-52.

Zaid, S.M. 2015. Geochemistry of sandstones from the Pliocene Gabir formation, north Marsa Alam, Red Sea, Egypt: Implication for provenance, weathering and tectonic setting. Journal of African Earth Sciences 102: 1-17.

Zaid, S.M., O. El-Badry, and A.M. Abdel-Fatah. 2017. Provenance of pharaonic potsherds, Sharkiya governorate, Egypt. Arabian Journal of Geosciences 10 (16): 354

Zaid, S.M., O. El-Badry, F. Ramadan, and M. Mohamed. 2015. Petrography and geochemistry of pharaonic sandstone monuments in tall san Al Hagr, A Sharqiya governorate, Egypt: Implications for provenance and tectonic setting. Turkish Journal of Earth Sciences 24 (4): 344-364.

\section{Submit your manuscript to a SpringerOpen ${ }^{\circ}$ journal and benefit from:}

- Convenient online submission

- Rigorous peer review

- Open access: articles freely available online

- High visibility within the field

Retaining the copyright to your article

Submit your next manuscript at $>$ springeropen.com 